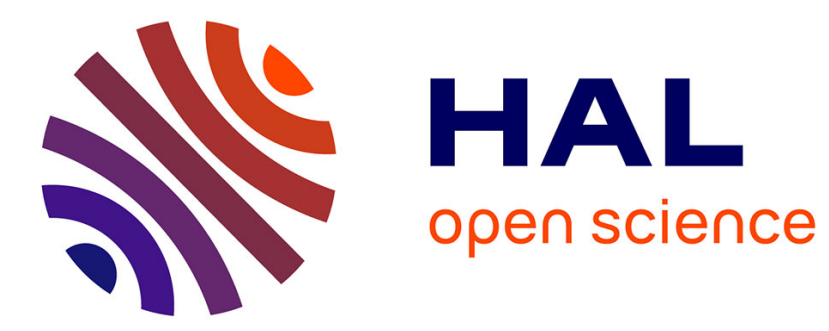

\title{
Static Elasticity in a Riemannian Manifold
}

Cristinel Mardare

\section{- To cite this version:}

Cristinel Mardare. Static Elasticity in a Riemannian Manifold. G-Q Chen, M. Grinfeld, R.J. Knops. Differential Geometry and Continuum Mechanics, pp.307-342, 2015. hal-01083499

\section{HAL Id: hal-01083499 \\ https://hal.sorbonne-universite.fr/hal-01083499}

Submitted on 17 Nov 2014

HAL is a multi-disciplinary open access archive for the deposit and dissemination of scientific research documents, whether they are published or not. The documents may come from teaching and research institutions in France or abroad, or from public or private research centers.
L'archive ouverte pluridisciplinaire HAL, est destinée au dépôt et à la diffusion de documents scientifiques de niveau recherche, publiés ou non, émanant des établissements d'enseignement et de recherche français ou étrangers, des laboratoires publics ou privés. 


\title{
Static Elasticity in a Riemannian Manifold
}

\author{
Cristinel Mardare
}

\section{Introduction}

This paper is adapted from Grubic, LeFloch, and Mardare [17]. The definitions and notations used, but not defined here, can be found in Section 2.

We discuss the deformation of an elastic body immersed in a Riemannian manifold in response to applied body and surface forces independent of time. We show how the equations of elastostatics can be derived from the principle of least energy, then prove that these equations possess a solution under explicit assumptions on the data. This result (Theorem 8) contains its counterparts in classical elasticity and holds under weaker assumptions. The proof relies on linearization around a natural state of the body and on Newton's method for finding zeroes of a mapping.

Our approach to the modeling of elastic bodies in a Riemannian manifold is a natural generalization of the classical theory of elasticity in the three-dimensional Euclidean space. Letting $(N, \hat{g})$ be the three-dimensional Euclidean space and $\varphi_{0}$ : $M \rightarrow \hat{M} \subset N$ be a global chart (under the assumption that it exists) of the reference configuration $\hat{M}:=\varphi_{0}(M)$ of an elastic body immersed in $N$ reduces our approach to the three-dimensional elasticity in curvilinear coordinates (see [11]), while letting $M=\hat{M} \subset N$ and $\varphi_{0}=\operatorname{id}_{\hat{M}}$ reduces our approach to the classical three-dimensional elasticity in Cartesian coordinates (see [10]).

Alternative approaches to the modeling of elastic bodies in a Riemannian manifold could be found elsewhere in the literature. A reference textbook is [19]; a relativistic approach to elasticity was initiated in [9], then developed in [3, 5, 6, 7, $8,15,27]$; a very general approach to modeling elastic bodies in manifolds without a metric was developed in [16, 21, 22, 23, 24].

A brief explanation of our notation is in order. It is important to keep in mind that the physical space containing the elastic body under consideration is a differential

Cristinel Mardare

Université Pierre et Marie Curie \& CNRS, Laboratoire Jacques-Louis Lions, Paris, F-75005

France, e-mail: mardare@ann.jussieu.fr 
manifold $N$ endowed with a single Riemannian metric $\hat{g}$, while the abstract configuration of the elastic body (by definition, a manifold whose points label the material points of the elastic body) is a differential manifold $M$ endowed with two metric tensors, one $g=g[\varphi]:=\varphi^{*} \hat{g}$ induced by an unknown deformation $\varphi: M \rightarrow N$, and one $g_{0}=g\left[\varphi_{0}\right]:=\varphi_{0}^{*} \hat{g}$ induced by a reference deformation $\varphi_{0}: M \rightarrow N$.

The connection and volume form induced on $N$ by $\hat{g}$ are denoted $\hat{\nabla}$ and $\hat{\boldsymbol{\omega}}$, respectively. The connection and volume form induced on $M$ by $g=g[\varphi]$ are denoted $\nabla=\nabla[\varphi]$ and $\boldsymbol{\omega}=\boldsymbol{\omega}[\varphi]$, while those induced on $M$ by $g_{0}=g\left[\varphi_{0}\right]$ are denoted $\nabla_{0}$ and $\boldsymbol{\omega}_{0}$. Note that $\nabla \boldsymbol{\omega}=0$ and $\nabla_{0} \boldsymbol{\omega}_{0}=0$.

Here and in the sequel, boldface letters denote $n$-forms with scalar or tensor coefficients; the corresponding plain letters denote components of such $n$-forms over a fixed volume form. For instance, if $W: M \rightarrow \mathbb{R}$ is a scalar function and $T: M \rightarrow$ $T_{1}^{1} M$ and $\hat{T}: N \rightarrow T_{1}^{1} N$ are tensor fields, then

$$
\boldsymbol{W}=W \boldsymbol{\omega}=W_{0} \boldsymbol{\omega}_{0} \text { and } \boldsymbol{T}=T \otimes \boldsymbol{\omega}=T_{0} \otimes \boldsymbol{\omega}_{0} \text { and } \hat{\boldsymbol{T}}=\hat{T} \otimes \hat{\boldsymbol{\omega}} .
$$

This notation, which is not used in classical elasticity, allows to do away with the Piola transform and use instead the more geometric pullback operator to define the stress tensor field (Section 4). Besides, it allows to write the boundary value problem of both nonlinear and linearized elasticity in divergence form (equations (2) and (3) below), by using appropriate volume forms: $\boldsymbol{\omega}$ in nonlinear elasticity and $\boldsymbol{\omega}_{0}$ in linearized elasticity.

Tensor fields on $M$ will be denoted by plain letters, such as $\xi$, and their components in a local chart will be denoted with Latin indices, such as $\xi^{i}$. Tensor fields on $N$ will be denoted by letters with a hat, such as $\hat{\xi}$, and their components in a local chart will be denoted with Greek indices, such as $\hat{\xi}^{\alpha}$.

Functionals defined over an infinite-dimensional manifold, such as $\mathscr{C}^{1}(M, N)$ or $\mathscr{C}^{1}(T M):=\left\{\xi: M \rightarrow T M ; \xi(x) \in T_{x} M\right\}$, will be denoted by letters with a bracket, such as $f[\cdot]$. Functions defined over a finite-dimensional manifold, such as $M$ or $T_{q}^{p} M$, will be denoted by letters with a paranthesis, such as $\dot{f}()$. Using the same letter in $f[]$ and $\dot{f}()$ means that the two functions are related, typically (but not always) by

$$
f[\varphi](x)=\dot{f}(x, \varphi(x), D \varphi(x)) \text { for all } x \in M,
$$

where $D \varphi(x)$ denotes the differential of $\varphi$ at $x$. In this case, the function $\dot{f}()$ is called the constitutive law of the function $f[]$ and the above relation is called the constitutive equation of $f$. Letters with several dots denote constitutive laws of different kind, e.g., at each $x \in M$,

$$
\begin{aligned}
\Sigma[\varphi](x) & =\dot{\Sigma}(x, \varphi(x), D \varphi(x)) & & \\
& =\ddot{\Sigma}(x, g[\varphi](x)), & & \text { where } g[\varphi]:=\varphi^{*} \hat{g}, \\
& =\dddot{\Sigma}\left(x, E\left[\varphi_{0}, \varphi\right](x)\right), & & \text { where } E\left[\varphi_{0}, \varphi\right]:=\frac{1}{2}\left(g[\varphi]-g \varphi_{0}\right), \\
& =\dddot{\Sigma}\left(x, \xi(x), \nabla_{0} \xi(x)\right), & & \text { where } \xi:=\exp _{\varphi_{0}}^{-1} \varphi,
\end{aligned}
$$


(the mapping $\exp _{\varphi_{0}}$ is defined below). The derivative of a function $f[]$ at a point $\varphi$ in the direction of a tangent vector $\eta$ at $\varphi$ will be denoted $f^{\prime}[\varphi] \eta$.

This paper is organized as follows. Section 2 specifies the mathematical framework and notation used throughout this paper.

Section 3 gathers the kinematic notions used to describe the deformation of an elastic body. The main novelty is the relation

$$
\varphi=\exp _{\varphi_{0}} \xi:=\left(\widehat{\exp }\left(\varphi_{0_{*}} \xi\right)\right) \circ \varphi_{0}
$$

between a displacement field $\xi \in \mathscr{C}^{1}(T M)$ of a reference configuration $\varphi_{0}(M)$ of the body and the corresponding deformation $\varphi: M \rightarrow N$ of the same body. Of course, this relation only holds if the vector field $\xi$ is small enough, so that the exponential maps of $N$ be well defined at each point $\varphi_{0}(x) \in N, x \in M$. Relation (1) plays a key role in the proof of Theorem 8 and replaces, to some extent, the missing vector space structure on the Riemann manifold $N$.

The metric tensor field associated with a deformation $\varphi: M \rightarrow N$, the strain tensor field associated with a reference deformation $\varphi: M \rightarrow N$ and a generic deformation $\psi: M \rightarrow N$, and the linearized strain tensor field associated with a reference deformation $\varphi: M \rightarrow N$ and a displacement field $\xi: M \rightarrow T M$, are respectively defined by

$$
g[\varphi]:=\varphi^{*} \hat{g}, \quad E[\varphi, \psi]:=\frac{1}{2}(g[\psi]-g[\varphi]), \text { and } e[\varphi, \xi]:=\frac{1}{2} \mathscr{L}_{\xi}(g[\varphi]) .
$$

Section 4 translates into mathematical terms the assumptions on the nature of the material constituting the body and of the applied body and surface forces. The assumption underlying our model is that the strain energy density associated with a deformation $\varphi$ of the body is of the form

$$
\boldsymbol{W}[\varphi](x):=\dddot{\boldsymbol{W}}\left(x, E\left[\varphi_{0}, \varphi\right](x)\right) \in \boldsymbol{\Lambda}_{x}^{n} M, x \in M,
$$

where $\varphi_{0}: M \rightarrow N$ denotes a reference deformation for which $\varphi_{0}(M) \subset N$ is a natural state (i.e., an unconstrained configuration) of the body. The stress tensor field associated with a deformation $\varphi$, and the elasticity tensor field, are then defined in terms of this density respectively by

$$
\boldsymbol{\Sigma}[\varphi]:=\frac{\partial \dddot{\boldsymbol{W}}}{\partial E}\left(\cdot, E\left[\varphi_{0}, \varphi\right]\right) \text { and } \boldsymbol{A}(x):=\frac{\partial^{2} \dddot{\boldsymbol{W}}}{\partial E^{2}}(x, 0), x \in M
$$

Other equivalent stress tensor fields, denoted $\boldsymbol{T}[\varphi], \tilde{\boldsymbol{T}}[\varphi], \hat{\boldsymbol{\Sigma}}[\varphi]$, and $\hat{\boldsymbol{T}}[\varphi]$, are defined in terms of $\boldsymbol{\Sigma}[\varphi]$ by lowering and/or pushing forward some of its indices.

Section 4 is also concerned with the modeling of applied body and surface forces. The main assumption is that the densities of these forces are of the form

$$
\begin{aligned}
& \boldsymbol{f}[\varphi](x):=\dot{\boldsymbol{f}}(x, \varphi(x), D \varphi(x)) \in T_{x}^{*} M \otimes \boldsymbol{\Lambda}_{x}^{n} M, \quad x \in M, \\
& \boldsymbol{h}[\varphi](x):=\dot{\boldsymbol{h}}(x, \varphi(x), D \varphi(x)) \in T_{x}^{*} M \otimes \boldsymbol{\Lambda}_{x}^{n-1} \Gamma_{2}, \quad x \in \Gamma_{2} \subset \partial M,
\end{aligned}
$$


where the functions $\dot{f}$ and $\dot{h}$ are sufficiently regular.

In Section 5, we state the equations of nonlinear elasticity in a Riemannian manifold first as a minimization problem (see (23)), then as variational equations (Theorem 2), and finally as a boundary value problem (Theorem 3 ). The latter asserts that the deformation $\varphi$ of the body must satisfy the system

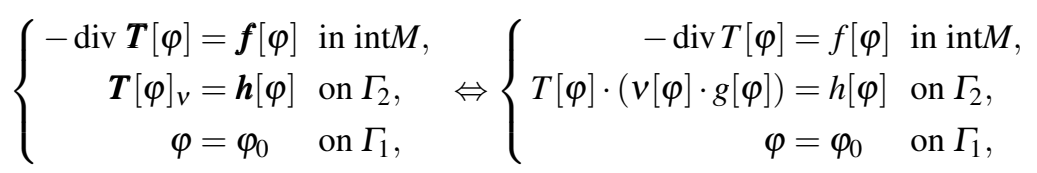

where $\operatorname{div}=\operatorname{div}[\varphi]$ and $v[\varphi]$ respectively denote the divergence operator and the unit outer normal vector field to the boundary of $M$ induced by the metric $g=g[\varphi]$, and where $\Gamma_{1} \cup \Gamma_{2}=\partial M$ denotes a partition of the boundary of $M$. Note that the divergence operators appearing in these boundary value problems depend themselves on the unknown $\varphi$.

In Section 6, we define the equations of linearized elasticity in a Riemannian manifold as the affine part of the equations of nonlinear elasticity with respect to the displacement field of a natural state of the body. Accordingly, the unknown displacement field $\xi \in \mathscr{C}^{1}(T M)$ satisfies the boundary value problem (see Theorem 5)

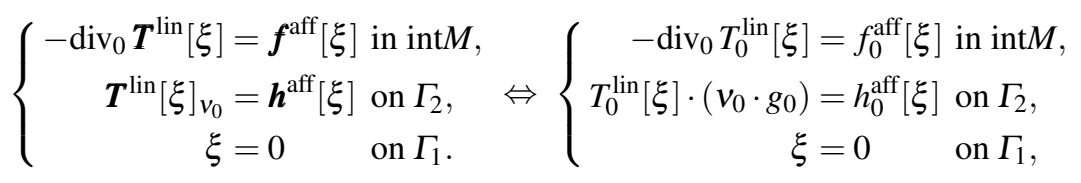

or equivalently, the variational equations

$$
\int_{M}\left(A: e\left[\varphi_{0}, \xi\right]\right): e\left[\varphi_{0}, \eta\right]=\int_{M} f^{\mathrm{aff}}[\xi] \cdot \eta+\int_{\Gamma_{2}} h^{\mathrm{aff}}[\xi] \cdot \eta
$$

for all sufficiently regular vector fields $\eta$ that vanish on $\Gamma_{1}$. Note that the divergence operator $\operatorname{div}_{0}$ appearing in (3) is independent of the unknown $\xi$, since it corresponds to the reference metric $g_{0}=g\left[\varphi_{0}\right]$.

In Section 7, we establish an existence and regularity theorem for the equations of linearized elasticity in a Riemannian manifold. We show that the variational equations (4) have a unique solution in the Sobolev space $\left\{\xi \in H^{1}(T M) ; \xi=0\right.$ on $\left.\Gamma_{1}\right\}$ provided the elasticity tensor field $\boldsymbol{A}$ is uniformly positive-definite and $\boldsymbol{f}^{\prime}\left[\varphi_{0}\right]$ and $\boldsymbol{h}^{\prime}\left[\varphi_{0}\right]$ are sufficiently small in an appropriate norm. The key to this existence result is a Riemannian version of Korn's inequality, due to [14], asserting that, if $\Gamma_{1} \neq \emptyset$, there exists a constant $C_{K}<\infty$ such that

$$
\|\xi\|_{H^{1}(T M)} \leq C_{K}\left\|\mathscr{L}_{\xi} g_{0}\right\|_{L^{2}\left(S_{2} M\right)}
$$

for all $\xi \in H^{1}(T M)$ that vanish on $\Gamma_{1}$. The smallness assumption mentioned above depends on this constant: the smaller $C_{K}$ is, the larger $\boldsymbol{f}^{\prime}\left[\varphi_{0}\right]$ and $\boldsymbol{h}^{\prime}\left[\varphi_{0}\right]$ are in the existence result for linearized elasticity. 
When $\Gamma_{1}=\partial M$, we show in addition that the solution to the equations of linearized elasticity belongs to the Sobolev space $W^{m+2, p}(T M), m \in \mathbb{N}, 1<p<\infty$, and satisfies the boundary value problem (3) if the data $\left(\partial M, \varphi_{0}, f\left[\varphi_{0}\right]\right.$, and $\left.f^{\prime}\left[\varphi_{0}\right]\right)$ satisfies specific regularity assumptions.

In Section 8, we study the existence of solutions to the equations of nonlinear elasticity (2) in the particular case where $\Gamma_{1}=\partial M$ and the constitutive laws of the elastic material and of the applied body forces are sufficiently regular. Under these assumptions, the equations of linearized elasticity define a surjective continuous linear operator $\mathscr{A}^{\operatorname{lin}}[\xi]:=\operatorname{div}_{0} T^{\operatorname{lin}}[\xi]+\boldsymbol{f}^{\prime}\left[\varphi_{0}\right] \xi: X \rightarrow \boldsymbol{Y}$, where

$$
X:=W^{m+2, p}(T M) \cap W_{0}^{1, p}(T M) \text { and } \boldsymbol{Y}:=W^{m, p}\left(T^{*} M \otimes \boldsymbol{\Lambda}^{n} M\right),
$$

for some exponents $m \in \mathbb{N}$ and $1<p<\infty$ that satisfy the constraint $(m+1) p>n$, where $n$ denotes the dimension of the manifold $M$.

Using the substitution $\varphi=\exp _{\varphi_{0}} \xi$, we recast the equations of nonlinear elasticity (2) into an equivalent (when $\xi$ is small enough so that the mapping $\exp _{\varphi_{0}}$ : $\mathscr{C}^{1}(T M) \rightarrow \mathscr{C}^{1}(M, N)$ be well-defined) boundary value problem, viz.,

$$
\begin{aligned}
-\operatorname{div} \boldsymbol{T}\left[\exp _{\varphi_{0}} \xi\right] & =\boldsymbol{f}\left[\exp _{\varphi_{0}} \xi\right] & & \text { in } \operatorname{int} M, \\
\xi & =0 & & \text { on } \partial M,
\end{aligned}
$$

whose unknown is the displacement field $\xi$. Then we show that the mapping $\mathscr{A}$ : $X \rightarrow \boldsymbol{Y}$ defined by

$$
\mathscr{A}[\xi]:=\operatorname{div} \boldsymbol{T}\left[\exp _{\varphi_{0}} \xi\right]+\boldsymbol{f}\left[\exp _{\varphi_{0}} \xi\right] \text { for all } \xi \in X,
$$

satisfies $\mathscr{A}^{\prime}[0]=\mathscr{A}^{\text {lin }}$. Thus proving an existence theorem for the equations of nonlinear elasticity amounts to proving the existence of a zero of the mapping $\mathscr{A}$. This is done by using a variant of Newton's method, where a zero of $\mathscr{A}$ is found as the limit of the sequence

$$
\xi_{1}:=0 \text { and } \xi_{k+1}:=\xi_{k}-\mathscr{A}^{\prime}[0]^{-1} \mathscr{A}\left[\xi_{k}\right], k \geq 1 .
$$

Note that the constraint $(m+1) p>n$ ensures that the Sobolev space $W^{m+1, p}\left(T_{1}^{1} M\right)$, to which $\nabla_{0} \xi$ belongs, is an algebra. This assumption is crucial in proving that the mapping $\mathscr{A}: X \rightarrow \boldsymbol{Y}$ is differentiable, since

$$
\mathscr{A}[\xi](x)=\dddot{\mathscr{A}}\left(x, \xi(x), \nabla_{0} \xi(x)\right), x \in M,
$$

for some regular enough mapping $\dddot{\mathscr{A}}$, defined in terms of the constitutive laws of the elastic material and of the applied forces under consideration; cf. relations (47) and (48). Thus $\mathscr{A}$ is a nonlinear Nemytskii (or substitution) operator, which is known to be non differentiable if $\xi$ belongs to a space with little regularity.

In addition to the regularity assumptions, we must assume that $f^{\prime}\left[\varphi_{0}\right]$ is sufficiently small in an appropriate norm, so that the operator $\mathscr{A}^{\prime}[0] \in \mathscr{L}(X, \boldsymbol{Y})$ is 
invertible; cf. Theorem 7 establishing the existence and regularity for linearized elasticity.

Finally, we point out that the assumptions of the existence theorem of Section 8 are slightly weaker than those usually made in classical elasticity, where either $p>n$ is imposed instead of $(m+1) p>n$ (cf. [10]), or $\dddot{\boldsymbol{f}}$ is assumed to belong to the smaller space $\mathscr{C}^{m+1}\left(M \times T M \times T_{1}^{1} M\right)$ (cf. [26]).

\section{Preliminaries}

For more details about the definitions below, see, e.g., [1] and [4].

Throughout this paper, $(N, \hat{g})$ denotes a smooth, oriented, Riemannian manifold of dimension $n$. $M$ denotes either a smooth, oriented, compact, differentiable manifold of dimension $n$, or $M=\bar{\Omega} \subset \tilde{M}$, where $\tilde{M}$ is a smooth, oriented, differentiable manifold of dimension $n$ and $\Omega$ is a bounded, connected, open subset of $\tilde{M}$, whose boundary $\Gamma:=\partial M$ is Lipschitz-continuous.

Generic points in $M$ and $N$ are denoted $x$ and $y$, respectively, or $\left(x^{i}\right)_{i=1}^{n}$ and $\left(y^{\alpha}\right)_{\alpha=1}^{n}$ in local coordinates. To ease notation, the $n$-tuples $\left(x^{i}\right)$ and $\left(y^{\alpha}\right)$ are also denoted $x$ and $y$, respectively.

The tangent and cotangent bundles of $M$ are denoted $T M:=\bigsqcup_{x \in M} T_{x} M$ and $T^{*} M:=\bigsqcup_{x \in M} T_{x}^{*} M$, respectively. The bundle of all $(p, q)$-tensors ( $p$-contravariant and $q$-covariant) is denoted $T_{q}^{p} M:=\left(\otimes^{p} T M\right) \otimes\left(\otimes^{q} T^{*} M\right)$. Partial contractions of one or two indices between two tensors will be denoted - or : , respectively.

The bundle of all symmetric $(0,2)$-tensors and the bundle of all symmetric $(2,0)$ tensors are respectively denoted

$$
S_{2} M:=\bigsqcup_{x \in M} S_{2, x} M \subset T_{2}^{0} M \text { and } S^{2} M:=\bigsqcup_{x \in M} S_{x}^{2} M \subset T_{0}^{2} M .
$$

The bundle of all positive-definite symmetric $(0,2)$-tensors is denoted $S_{2}^{+} M:=$ $\bigsqcup_{x \in M} S_{2, x}^{+} M \subset S_{2} M$.

The bundle of all $k$-forms (that is, totally antisymmetric $(0, k)$-tensors fields) is denoted $\Lambda^{k} M:=\bigsqcup_{x \in M} \Lambda_{x}^{k} M$; volume forms (that is, $n$-forms on $M$ and $(n-1)$-forms on the boundary of $M$ ) will be denoted by boldface letters, such as $\boldsymbol{\omega}$ and $\boldsymbol{i}_{v} \boldsymbol{\omega}$.

Fiber bundles on $M \times N$ will also be used with self-explanatory notation. For instance,

$$
T^{*} M \otimes T N:=\bigsqcup_{(x, y) \in M \times N} T_{x}^{*} M \otimes T_{y} N,
$$

where $T_{x}^{*} M \otimes T_{y} N$ is canonically identified with the space $\mathscr{L}\left(T_{x} M, T_{y} N\right)$ of all linear mappings from $T_{x} M$ to $T_{y} N$.

The set of all mappings $\varphi: M \rightarrow N$ of class $\mathscr{C}^{k}$ is denoted $\mathscr{C}^{k}(M, N)$. Given any mapping $\varphi \in \mathscr{C}^{0}(M, N)$, the pullback bundle of $T_{q}^{p} N$ by $\varphi$ is denoted and defined by 


$$
\varphi^{*} T_{q}^{p} N:=\bigsqcup_{x \in M} T_{q, \varphi(x)}^{p} N .
$$

The pushforward and pullback mappings are denoted $\varphi_{*}: T_{0}^{p} M \rightarrow T_{0}^{p} N$ and $\varphi^{*}$ : $T_{q}^{0} M \rightarrow T_{q}^{0} N$, respectively. For instance, if $p=1$ and $q=2$, then, at each $x \in M$,

$$
\left(\varphi_{*} \xi\right)^{\alpha}(\varphi(x)):=\frac{\partial \varphi^{\alpha}}{\partial x^{i}}(x) \xi^{i}(x) \text { and }\left(\varphi^{*} \hat{g}_{i j}(x):=\frac{\partial \varphi^{\alpha}}{\partial x^{i}}(x) \frac{\partial \varphi^{\beta}}{\partial x^{j}}(x) \hat{g}_{\alpha \beta}(\varphi(x))\right.
$$

where the functions $y^{\alpha}=\varphi^{\alpha}\left(x^{i}\right)$ describe the mapping $\varphi$ in local coordinates $\left(x^{i}\right)$ on $M$ and $\left(y^{\alpha}\right)$ on $N$.

The Lie derivative operators on $M$ and $N$ are respectively denoted $\mathscr{L}$ and $\tilde{\mathscr{L}}$. For instance, the Lie derivative of $\hat{g}$ along a vector field $\hat{\xi} \in \mathscr{C}^{1}(T N)$ is defined by

$$
\hat{\mathscr{L}}_{\hat{\xi}} \hat{g}:=\lim _{t \rightarrow 0} \frac{1}{t}\left(\gamma_{\hat{\xi}}(\cdot, t)^{*} \hat{g}-\hat{g}\right)
$$

where $\gamma_{\hat{\xi}}$ denotes the flow of $\hat{\xi}$. This flow is defined by $(y, t) \in(-\varepsilon, \varepsilon) \rightarrow \gamma_{\hat{\xi}}(y, t) \in$ $N$, where $\gamma_{\hat{\xi}}(y, \cdot)$ is the unique solution to the Cauchy problem

$$
\gamma_{\hat{\xi}}(y, 0)=y \text { and } \frac{d}{d t} \gamma_{\hat{\xi}}(y, t)=\hat{\xi}\left(\gamma_{\hat{\xi}}(y, t)\right) \text { for all } t \in(-\varepsilon, \varepsilon),
$$

where $\varepsilon>0$ denotes a small enough parameter (whose existence follows from the compactness of $M$ ).

The notation $\left.\xi\right|_{\Gamma}$ designates the restriction to the set $\Gamma$ of a function or a tensor field $\xi$ defined over a set that contains $\Gamma$. Given any smooth fiber bundle $X$ over $M$ and any submanifold $\Gamma \subset M$, we denote by $\mathscr{C}^{k}(X)$ the space of all sections of class $\mathscr{C}^{k}$ of the fiber bundle $X$, and we let

$$
\mathscr{C}^{k}\left(\left.X\right|_{\Gamma}\right):=\left\{\left.S\right|_{\Gamma} ; S \in \mathscr{C}^{k}(X)\right\} .
$$

If $S \in \mathscr{C}^{k}(X)$ is a section of a fiber bundle $X$ over $M$, then $S(x)$ denotes the value of $S$ at $x \in M$.

The tangent at $x \in M$ of a mapping $\varphi \in \mathscr{C}^{k}(M, N)$ is a linear mapping $T_{x} \varphi \in$ $\mathscr{L}\left(T_{x} M, T_{\varphi(x)} N\right)$. The section $D \varphi \in \mathscr{C}^{k-1}\left(T^{*} M \otimes \varphi^{*} T N\right)$, defined at each $x \in M$ by

$$
D \varphi(x) \cdot \xi(x):=\left(T_{x} \varphi\right)(\xi(x)) \text { for all } \xi \in T M,
$$

is the differential of $\varphi$ at $x$. In local charts,

$$
D \varphi(x)=\frac{\partial \varphi^{\alpha}}{\partial x^{i}}(x) d x^{i}(x) \otimes \frac{\partial}{\partial y^{\alpha}}(\varphi(x)), x \in M .
$$

Let $\hat{\nabla}: \mathscr{C}^{k}(T N) \rightarrow \mathscr{C}^{k-1}\left(T^{*} N \otimes T N\right)$ denote the Levi-Civita connection on the Riemannian manifold $N$ induced by the metric $\hat{g}$, defined in local coordinates by 


$$
\hat{\nabla}_{\alpha} \hat{\xi}^{\beta}=\frac{\partial \hat{\xi}^{\beta}}{\partial y^{\alpha}}+\hat{\Gamma}_{\alpha \gamma}^{\beta} \hat{\xi}^{\gamma}
$$

where $\hat{\Gamma}_{\alpha \gamma}^{\beta}$ denote the Christoffel symbols associated with the metric tensors $\hat{g}$. The connection $\hat{\nabla}$ is extended to arbitrary tensor fields on $N$ in the usual manner, by using the Leibnitz rule.

Any immersion $\varphi \in \mathscr{C}^{k+1}(M, N)$ induces on $M$ the metric tensor field

$$
g=g[\varphi]:=\varphi^{*} \hat{g} \in \mathscr{C}^{k}\left(S_{2}^{+} M\right),
$$

and the Levi-Civita connection associated with $g=g[\varphi]$

$$
\nabla=\nabla[\varphi]: \mathscr{C}^{k}(T M) \rightarrow \mathscr{C}^{k-1}\left(T^{*} M \otimes T M\right) .
$$

In local coordinates, we have

$$
g_{i j}=\frac{\partial \varphi^{\alpha}}{\partial x^{i}} \frac{\partial \varphi^{\beta}}{\partial x^{j}}\left(\hat{g}_{\alpha \beta} \circ \varphi\right) \text { and } \nabla_{i} \xi^{j}=\frac{\partial \xi^{j}}{\partial x^{i}}+\Gamma_{i k}^{j} \xi^{k},
$$

where $\Gamma_{i k}^{j}$ denote the Christoffel symbols associated with the metric $g$. Note that the metric $g=g[\varphi]$ and connection $\nabla=\nabla[\varphi]$ depend on the immersion $\varphi$.

The divergence operators induced by the connections $\nabla=\nabla[\varphi]$ and $\hat{\nabla}$ are respectively denoted $\operatorname{div}=\operatorname{div}[\varphi]$ and $\widehat{\operatorname{div}}$. If $\boldsymbol{T}=T \otimes \boldsymbol{\omega}$ with $T \in \mathscr{C}^{1}\left(T M \otimes T^{*} M\right)$ and $\boldsymbol{\omega} \in \boldsymbol{\Lambda}^{n} M$, then

$$
\begin{aligned}
\operatorname{div} T & :=\left(\nabla_{i} T_{k}^{i}\right) d x^{k}, \\
\operatorname{div} \boldsymbol{T} & :=\left(\nabla_{i} \boldsymbol{T}_{j_{1} \ldots j_{n} k}^{i}\right) d x^{j_{1}} \otimes \ldots \otimes d x^{j_{n}} \otimes d x^{k} .
\end{aligned}
$$

Note that if the volume form satisfies $\nabla \boldsymbol{\omega}=0$, then

$$
\operatorname{div} \boldsymbol{T}=(\operatorname{div} T) \otimes \boldsymbol{\omega} \text { and } \nabla_{\eta} \boldsymbol{T}=\left(\nabla_{\eta} T\right) \otimes \boldsymbol{\omega} \text { for all } \eta \in \mathscr{C}^{0}(T M) .
$$

The interior product $\boldsymbol{i}_{\eta}: \boldsymbol{T} \in \mathscr{C}^{0}\left(\boldsymbol{\Lambda}^{n} M \otimes T M \otimes T^{*} M\right) \rightarrow \boldsymbol{i}_{\eta} \boldsymbol{T} \in \mathscr{C}^{0}\left(\boldsymbol{\Lambda}^{n-1} M \otimes\right.$ $\left.T M \otimes T^{*} M\right)$ is defined by

$$
\left(\boldsymbol{i}_{\eta} \boldsymbol{T}\right)\left(\zeta_{1}, \ldots, \zeta_{n-1} ; \theta, \xi\right):=\boldsymbol{T}\left(\eta, \zeta_{1}, \ldots, \zeta_{n-1} ; \theta, \xi\right)
$$

for all $\eta, \zeta_{1}, \ldots, \zeta_{n-1} \in \mathscr{C}^{0}(T M), \theta \in \mathscr{C}^{0}\left(T^{*} M\right)$, and $\xi \in \mathscr{C}^{0}(T M)$, or equivalently by

$$
\boldsymbol{i}_{\eta} \boldsymbol{T}=T \otimes \boldsymbol{i}_{\eta} \boldsymbol{\omega} \text { if } \boldsymbol{T}=T \otimes \boldsymbol{\omega} .
$$

The normal trace of a tensor field $\boldsymbol{T} \in \mathscr{C}^{0}\left(\boldsymbol{\Lambda}^{n} M \otimes T M \otimes T^{*} M\right)$ on the boundary $\partial M$ is defined by

$$
\boldsymbol{T}_{v}:=\left(\boldsymbol{i}_{v} \boldsymbol{T}\right) \cdot(v \cdot g) \in \mathscr{C}^{0}\left(\boldsymbol{\Lambda}^{n-1}(\partial M) \otimes T^{*} M\right),
$$

or equivalently, by 


$$
\boldsymbol{T}_{v}=(T \cdot(v \cdot g)) \otimes \boldsymbol{i}_{v} \boldsymbol{\omega} \text { if } \boldsymbol{T}=T \otimes \boldsymbol{\omega},
$$

where $v$ denotes the unit outer normal vector field to $\partial M$ defined by the metric $g$. Note that the definition of $\boldsymbol{T}_{v}$ is independent of the choice of the Riemannian metric $g$, since

$$
\left(\boldsymbol{i}_{v_{1}} \boldsymbol{T}\right) \cdot\left(v_{1} \cdot g_{1}\right)=\left(\boldsymbol{i}_{v_{2}} \boldsymbol{T}\right) \cdot\left(v_{2} \cdot g_{2}\right)
$$

for all Riemannian metrics $g_{1}$ and $g_{2}$ on $M\left(v_{i}\right.$ denotes the unit outer normal vector field to $\partial M$ defined by the metric $\left.g_{i}, i=1,2\right)$. Indeed,

$$
\left(\boldsymbol{i}_{v_{1}} \boldsymbol{T}\right) \cdot\left(v_{1} \cdot g_{1}\right)=g_{2}\left(v_{1}, v_{2}\right)\left[\left(\boldsymbol{i}_{v_{2}} \boldsymbol{T}\right) \cdot\left(v_{1} \cdot g_{1}\right)\right]
$$

and

$$
g_{2}\left(v_{1}, v_{2}\right)\left(v_{1} \cdot g_{1}\right)=v_{2} \cdot g_{2} .
$$

The above definition of the normal trace is justified by the following integration by parts formula, the proof of which is classical. Recall that ·, respectively :, denotes the contraction of one, respectively two, indices.

Lemma 1. Let $\xi \in \mathscr{C}^{1}(T M)$ and $\boldsymbol{T}=T \otimes \boldsymbol{\omega} \in \mathscr{C}^{1}\left(T_{1}^{1} M \otimes \boldsymbol{\Lambda}^{n} M\right)$, where $\boldsymbol{\omega} \in \boldsymbol{\Lambda}^{n} M$ satisfies $\nabla \boldsymbol{\omega}=0$. Then

$$
\int_{M} \boldsymbol{T}: \nabla \xi=-\int_{M}(\operatorname{div} \boldsymbol{T}) \cdot \xi+\int_{\partial M} \boldsymbol{T}_{v} \cdot \xi
$$

where $\boldsymbol{T}: \nabla \xi \stackrel{\text { def }}{=}(T: \nabla \xi) \boldsymbol{\omega},(\operatorname{div} \boldsymbol{T}) \cdot \boldsymbol{\xi}=((\operatorname{div} T) \cdot \xi) \boldsymbol{\omega}$, and $\boldsymbol{T}_{v} \cdot \boldsymbol{\xi}=((T \cdot(\nu \cdot g))$. $\xi) i_{V} \omega$.

All functions and tensor fields appearing in Sections 3-6 are of class $\mathscr{C}^{k}$ over their domain of definition, with $k$ sufficiently large so that all differential operators be defined in the classical sense (as opposed to the distributional sense). Functions and tensor fields belonging to Sobolev spaces on the Riemannian manifold $\left(M, g_{0}\right)$ will be used in Sections 7-8 in order to prove existence theorems for the models introduced in Sections 5 and 6. Following [4], the Sobolev space $W^{k, p}(T M)$ is defined for each $k \in \mathbb{N}$ and $1 \leq p<\infty$ as the completion in the Lebesgue space $L^{p}(T M)$ of the space $\mathscr{C}^{k}(T M)$ with respect to the norm

$$
\|\xi\|_{k, p}=\|\xi\|_{W^{k, p}(T M)}:=\left\{\int_{M}\left(|\xi|^{p}+\sum_{\ell=1}^{k}\left|\nabla_{0}^{\ell} \xi\right|^{p}\right) \boldsymbol{\omega}_{0}\right\}^{1 / p}
$$

where

$$
\begin{aligned}
\left|\nabla_{0}^{\ell} \xi\right| & :=\left\{g_{0}\left(\nabla_{0}^{\ell} \xi, \nabla_{0}^{\ell} \xi\right)\right\}^{1 / 2} \\
& =\left\{\left(g_{0}\right)_{i j}\left(g_{0}\right)^{i_{1} j_{1}} \ldots\left(g_{0}\right)^{i_{\ell} j_{\ell}}\left(\nabla_{0}\right)_{i_{1} \ldots i_{\ell}} \xi^{i}\left(\nabla_{0}\right)_{j_{1} \ldots j_{\ell}} \xi^{j}\right\}^{1 / 2} .
\end{aligned}
$$

The Sobolev space $W_{0}^{k, p}(T M)$ is defined as the closure in $W^{k, p}(T M)$ of the space 


$$
\mathscr{C}_{c}^{k}(T M):=\left\{\xi \in \mathscr{C}^{k}(T M) ; \overline{\{x \in M ; \xi(x) \neq 0\}} \subset \operatorname{int} M\right\} .
$$

We will also use the notation $H^{k}(T M):=W^{k, 2}(T M)$ and $H_{0}^{k}(T M):=W_{0}^{k, 2}(T M)$.

\section{Kinematics}

The kinematic notions introduced below are natural extensions of their counterparts in classical elasticity. More specifically, if the Riemannian manifold $(N, \hat{g})$ appearing below is the three-dimensional Euclidean space and if the reference configuration of the elastic body is described by a single local chart with $M$ as its domain of definition, then our definitions coincide with the classical ones in curvilinear coordinates (see, e.g., [11]).

Consider an elastic body with abstract configuration $M$ undergoing a deformation in a Riemannian manifold $(N, \hat{g})$ in response to applied body and surface forces.

A deformation of the body is an immersion $\varphi \in \mathscr{C}^{1}(M, N)$ that preserves orientation and satisfies the axiom of impenetrability of matter. This means that

$$
\begin{aligned}
& \operatorname{det} D \varphi(x)>0 \text { for all } x \in M, \\
& \left.\varphi\right|_{\operatorname{int} M}: \operatorname{int} M \rightarrow N \text { is injective, }
\end{aligned}
$$

where int $M$ denotes the interior of $M$. Note that $\varphi$ needs not be injective on the whole $M$ since self-contact of the deformed boundary may occur. The set of all deformations is denoted

$$
\operatorname{Def}(M, N):=\left\{\varphi \in \mathscr{C}^{1}(M, N) ;\left.\varphi\right|_{\text {int } M} \text { injective, } \operatorname{det} D \varphi>0 \text { in } M\right\} .
$$

An admissible deformation of the body is a deformation that satisfies the Dirichlet boundary condition

$$
\varphi=\varphi_{0} \text { on } \Gamma_{1}
$$

on a (possibly empty) portion $\Gamma_{1} \subset \Gamma:=\partial M$ of the boundary of $M$. The immersion $\varphi_{0} \in \mathscr{C}^{1}(M, N)$ specifies the position of the points of the elastic body that are kept fixed.

A displacement field of the body associated with a given deformation $\varphi \in$ $\mathscr{C}^{1}(M, N)$ is a section $\tilde{\xi} \in \mathscr{C}^{1}\left(\varphi^{*} T N\right)$. If the deformation $\varphi$ is of class $\mathscr{C}^{2}$, then each displacement field $\tilde{\xi} \in \mathscr{C}^{1}\left(\varphi^{*} T N\right)$ is induced by a vector field $\xi \in \mathscr{C}^{1}(T M)$ by means of the bijective mapping

$$
\xi \rightarrow \tilde{\xi}:=\left(\varphi_{*} \xi\right) \circ \varphi
$$

Let $\hat{\delta}(y)$ denote the injectivity radius of $N$ at $y \in N$, let $\hat{\delta}(\varphi(M)):=\min _{y \in \varphi(M)} \hat{\delta}(y)$ denote the injectivity radius of the compact subset $\varphi(M)$ of $N$, let

$$
\mathscr{C}_{\varphi}^{0}(T M):=\left\{\xi \in \mathscr{C}^{0}(T M) ;\left\|\varphi_{*} \xi\right\|_{\mathscr{C}^{0}\left(\left.T N\right|_{\varphi(M)}\right)}<\hat{\delta}(\varphi(M))\right\}
$$


and let $\widehat{\exp }$ denote the exponential maps on $N$. It is clear that the mapping

$$
\exp _{\varphi}:=\widehat{\exp } \circ D \varphi: \mathscr{C}_{\varphi}^{0}(T M) \rightarrow \mathscr{C}^{0}(M, N)
$$

is a $\mathscr{C}^{1}$-diffeomorphism onto its image. Therefore any deformation $\psi \in \mathscr{C}^{0}(M, N)$ that is close in the $\mathscr{C}^{0}$-norm to a given deformation $\varphi \in \mathscr{C}^{1}(M, N)$ can be written in an unique manner as

$$
\psi=\exp _{\varphi} \xi:=\left(\widehat{\exp } \varphi_{*} \xi\right) \circ \varphi .
$$

This observation will be used in Sections 6-8 to transform the equations of elasticity in which the unknown is the deformation $\varphi$, assumed to be close in the $\mathscr{C}^{0}$-norm to a reference deformation $\varphi_{0}$, into equivalent equations in which the unknown is the displacement field $\xi:=\exp _{\varphi_{0}}^{-1} \varphi$, where $\exp _{\varphi_{0}}^{-1}$ denotes the inverse of the diffeomorphism $\exp _{\varphi_{0}}$.

Remark 1. (a) The relation $\psi=\exp _{\varphi} \xi$ means that, for each $x \in M, \psi(x)$ is the end-point of the geodesic arc in $N$ with length $|\xi(x)|$ starting at the point $\varphi(x)$ in the direction of $\left(\varphi_{*} \xi\right)(\varphi(x))$.

(b) The relation $\xi=\exp _{\varphi}^{-1} \psi$ means that, for each $x \in M, \xi(x)$ is the pullback by $\varphi$ of the vector that is tangent at $\varphi(x)$ to the geodesic arc joining $\varphi(x)$ to $\psi(x)$ in $N$ and whose norm equals the length of this geodesic arc.

The metric tensor field, also called the right Cauchy-Green tensor field, associated with a deformation $\varphi \in \mathscr{C}^{1}(M, N)$ is the pullback by $\varphi$ of the metric $\hat{g}$ of $N$, i.e.,

$$
g[\varphi]:=\varphi^{*} \hat{g} .
$$

Note that the notation $C:=g[\varphi]$ is often used in classical elasticity.

The strain tensor field, also called Green-St Venant tensor field, associated with two deformations $\varphi, \psi \in \mathscr{C}^{1}(M, N)$ is defined by

$$
E[\varphi, \psi]:=\frac{1}{2}(g[\varphi]-g[\psi]) .
$$

The first argument $\varphi$ is considered as a deformation of reference, while the second argument $\psi$ is an arbitrary deformation.

The linearized, or infinitesimal, strain tensor field associated with a deformation $\varphi \in \mathscr{C}^{1}(M, N)$ and a displacement field $\xi \in \mathscr{C}^{1}(T M)$ of the set $\varphi(M)$ is the linear part with respect to $\xi$ of the mapping $\xi \mapsto E\left[\varphi, \exp _{\varphi} \xi\right]$, i.e.,

$$
e[\varphi, \xi]:=\left[\frac{d}{d t} E\left[\varphi, \exp _{\varphi}(t \xi)\right]\right]_{t=0} .
$$

Explicit expressions of $e[\varphi, \xi]$ are given in Theorem 1 below, first in terms of the Lie derivative and connection on $M$, then in terms of the Lie derivative and connection on $N$. Recall that - denotes the partial contraction of one single index of two tensors.

Theorem 1. Let $\varphi \in \mathscr{C}^{1}(M, N)$ be an immersion and let $\xi \in \mathscr{C}^{1}(T M)$ be a vector field on $M$. Then 
(a)

$$
2 e[\varphi, \xi]=\mathscr{L}_{\xi} g=\nabla \xi^{b}+\left(\nabla \xi^{b}\right)^{T}=g \cdot \nabla \xi+(g \cdot \nabla \xi)^{T}
$$

where $\mathscr{L}$ denotes the Lie derivative operator on $M, g:=\varphi^{*} \hat{g}, \nabla$ denotes the connection on $M$ induced by the metric $g$, and $\xi^{b}:=g \cdot \xi$. In local charts, these equations read

$$
2 e_{i j}[\varphi, \xi]=\nabla_{i} \xi_{j}+\nabla_{j} \xi_{i}=g_{j k} \nabla_{i} \xi^{k}+g_{i k} \nabla_{j} \xi^{k},
$$

where $\xi(x)=\xi_{i}(x) d x^{i}(x)$ and $e[\varphi, \xi]=e_{i j}[\varphi, \xi] d x^{i}(x) \otimes d x^{j}(x), x \in M$.

(b)

$$
e[\varphi, \xi]=\varphi^{*}(\hat{e}[\hat{\xi}]), \quad \hat{\xi}:=\varphi_{*} \xi
$$

where

$$
2 \hat{e}[\hat{\xi}]:=\hat{\mathscr{L}}_{\hat{\xi}} \hat{g}=\hat{\nabla} \hat{\xi}^{b}+\left(\hat{\nabla} \hat{\xi}^{b}\right)^{T}=\hat{g} \cdot \hat{\nabla} \hat{\xi}+(\hat{g} \cdot \hat{\nabla} \hat{\xi})^{T}
$$

$\hat{\mathscr{L}}$ denotes the Lie derivative operator on $N, \hat{g}$ is the metric on $N, \hat{\nabla}$ denotes the connection induced by $\hat{g}$, and $\hat{\xi}^{b}:=\hat{g} \cdot \hat{\xi}$. In local charts,

$$
2 e_{i j}[\varphi, \xi]=\frac{\partial \varphi^{\alpha}}{\partial x^{i}} \frac{\partial \varphi^{\beta}}{\partial x^{j}}\left(\hat{\nabla}_{\beta} \hat{\xi}_{\alpha}+\hat{\nabla}_{\alpha} \hat{\xi}_{\beta}\right) \circ \varphi, \text { where } \hat{\xi}(y)=\hat{\xi}_{\alpha}(y) d y^{\alpha}(y) \text {. }
$$

Proof. For each $t$ in a neighborhood of zero, define the deformations

$$
\varphi(\cdot, t):=\exp _{\varphi}(t \xi) \text { and } \psi(\cdot, t):=\gamma_{\hat{\xi}}(\cdot, t) \circ \varphi
$$

where $\hat{\xi} \in \mathscr{C}^{1}(T N)$ denotes any extension of the section $\varphi_{*} \xi \in \mathscr{C}^{1}(T \varphi(M))$ and $\gamma_{\hat{\xi}}$ denotes the flow of $\hat{\xi}$ (see Section 2 ). By definition,

$$
e[\varphi, \xi]=\left[\frac{d}{d t} E[\varphi, \varphi(\cdot, t)]\right]_{t=0}=\lim _{t \rightarrow 0} \frac{\varphi(\cdot, t)^{*} \hat{g}-\varphi^{*} \hat{g}}{2 t} .
$$

Since

$$
\frac{\partial \varphi}{\partial t}(x, 0)=\frac{\partial \psi}{\partial t}(x, 0)=\xi(x) \text { for all } x \in M,
$$

it follows from the above expression of $e[\varphi, \xi]$ that

$$
2 e[\varphi, \xi]=\lim _{t \rightarrow 0} \frac{\psi(\cdot, t)^{*} \hat{g}-\varphi^{*} \hat{g}}{t} .
$$

Then the definition of the Lie derivative yields

$$
\begin{aligned}
2 e[\varphi, \xi] & =\varphi^{*}\left(\lim _{t \rightarrow 0} \frac{\gamma_{\hat{\xi}}(\cdot, t)^{*} \hat{g}-\hat{g}}{t}\right)=\varphi^{*}\left(\hat{\mathscr{L}}_{\hat{\xi}} \hat{g}\right) \\
& =\varphi^{*}\left(\hat{\mathscr{L}}_{\varphi_{*}} \xi \hat{g}\right)=\mathscr{L}_{\xi}\left(\varphi^{*} \hat{g}\right)=\mathscr{L}_{\xi} g .
\end{aligned}
$$

Expressing the Lie derivative $\hat{\mathscr{L}}_{\hat{\xi}} \hat{g}$ in terms of the connection $\hat{\nabla}$ gives 


$$
\begin{aligned}
2 e_{i j}[\varphi, \xi] & =\frac{\partial \varphi^{\alpha}}{\partial x^{i}} \frac{\partial \varphi^{\beta}}{\partial x^{j}}\left(\hat{g}_{\alpha \gamma} \hat{\nabla}_{\beta} \hat{\xi}^{\gamma}+\hat{g}_{\beta \gamma} \hat{\nabla}_{\alpha} \hat{\xi}^{\gamma}\right) \circ \varphi \\
& =\frac{\partial \varphi^{\alpha}}{\partial x^{i}} \frac{\partial \varphi^{\beta}}{\partial x^{j}}\left(\hat{\nabla}_{\beta} \hat{\xi}_{\alpha}+\hat{\nabla}_{\alpha} \hat{\xi}_{\beta}\right) \circ \varphi,
\end{aligned}
$$

which implies in turn that

$$
e_{i j}[\varphi, \xi]=\frac{1}{2}\left(g_{i k} \nabla_{j} \xi^{k}+g_{j k} \nabla_{i} \xi^{k}\right)=\frac{1}{2}\left(\nabla_{j} \xi_{i}+\nabla_{j} \xi_{j}\right) .
$$

\section{Elastic materials and applied forces}

More details about the definitions below can be found in [17].

Consider an elastic body with abstract configuration $M$ subjected to applied body and surface forces in a Riemannian manifold $(N, \hat{g})$.

Let a reference deformation $\varphi_{0} \in \mathscr{C}^{3}(M, N)$ be given in such a way that the configuration $\varphi_{0}(M) \subset N$ of the elastic body be a natural state (i.e., unconstrained). Let $g_{0}:=\varphi_{0}^{*} \hat{g}$ and $\boldsymbol{\omega}_{0}:=\varphi_{0}^{*} \hat{\boldsymbol{\omega}}$ respectively denote the metric tensor field and the volume form on $M$ induced by $\varphi_{0}$.

In all that follows, the stored energy function of the elastic material constituting the body is defined, at each $x \in M$, by the mappings

$$
\dddot{\boldsymbol{W}}(x, \cdot)=\dddot{W}_{0}(x, \cdot) \boldsymbol{\omega}_{0}(x): S_{2, x} M \rightarrow \boldsymbol{\Lambda}_{x}^{n} M .
$$

We assume without loss of generality that $\dddot{\boldsymbol{W}}(x, 0)=0$.

Remark 2. An example of stored energy function is that of Saint Venant - Kirchhoff, viz.,

$$
\dddot{\boldsymbol{W}}_{s v k}(x, E):=\left(\frac{\lambda}{2}(\operatorname{tr} E)^{2}+\mu|E|^{2}\right) \boldsymbol{\omega}_{0}(x)
$$

for all $x \in M$ and all $E \in S_{2, x} M$, where $\operatorname{tr} E:=g_{0}{ }^{i j} E_{i j}$ and $|E|^{2}:=g_{0}{ }^{i k} g_{0}{ }^{j \ell} E_{k \ell} E_{i j}$. The two scalar parameters $\lambda \geq 0$ and $\mu>0$ are called the Lamé constants of the elastic material.

Let the Gateaux derivative of the mapping $\dddot{\boldsymbol{W}}(x, \cdot): S_{2, x} M \rightarrow \boldsymbol{\Lambda}_{x}^{n} M$ at $E \in S_{2, x} M$ in the direction $H \in S_{2, x} M$ be defined by

$$
\frac{\partial \dddot{\boldsymbol{W}}}{\partial E}(x, E): H=\lim _{t \rightarrow 0} \frac{1}{t}\{\dddot{\boldsymbol{W}}(x, E+t H)-\dddot{\boldsymbol{W}}(x, E)\} .
$$

The constitutive law of an elastic material whose stored energy function is $\dddot{W} \in$ $\mathscr{C}^{1}\left(S_{2} M, \Lambda^{n} M\right)$ is the mapping associating to each $x \in M$ and each $E \in S_{2, x} M$ the tensor

$$
\dddot{\boldsymbol{\Sigma}}(x, E)=\dddot{\Sigma}_{0}(x, E) \otimes \boldsymbol{\omega}_{0}(x):=\frac{\partial \dddot{\boldsymbol{W}}}{\partial E}(x, E)=\frac{\partial \dddot{W}_{0}}{\partial E}(x, E) \otimes \boldsymbol{\omega}_{0}(x)
$$


in $S_{x}^{2} M \otimes \boldsymbol{\Lambda}_{x}^{n} M$. The assumption that the reference configuration $\varphi_{0}(M) \subset N$ is a natural state means that $\dddot{\boldsymbol{\Sigma}}(x, 0)=0$.

The elasticity tensor field of an elastic material whose stored energy function is $\dddot{\boldsymbol{W}} \in \mathscr{C}^{2}\left(S_{2} M \otimes \boldsymbol{\Lambda}^{n} M\right)$ is the section $\boldsymbol{A}=A_{0} \otimes \boldsymbol{\omega}_{0}, A_{0} \in \mathscr{C}^{1}\left(S^{2} M \otimes_{\text {sym }} S^{2} M\right)$, defined at each $x \in M$ by

$$
\boldsymbol{A}(x):=\frac{\partial^{2} \dddot{\boldsymbol{W}}}{\partial E^{2}}(x, 0) \Leftrightarrow A_{0}(x):=\frac{\partial^{2} \dddot{W}_{0}}{\partial E^{2}}(x, 0) .
$$

Note that the components of $A_{0}$ in a local chart satisfy the symmetries

$$
A_{0}{ }^{i j k \ell}=A_{0}{ }^{k \ell i j}=A_{0}{ }^{j i k \ell}=A_{0}{ }^{i j \ell k} .
$$

The strain energy corresponding to a deformation $\varphi \in \operatorname{Def}(M, N)$ of the elastic body under consideration is defined by

$$
I[\varphi]:=\int_{M} W[\varphi]
$$

where, at each $x \in M$,

$$
\boldsymbol{W}[\varphi](x)=\dddot{\boldsymbol{W}}\left(x, E\left[\varphi_{0}, \varphi\right](x)\right) .
$$

Recall that $E\left[\varphi_{0}, \varphi\right]:=\frac{1}{2}\left(\varphi^{*} \hat{g}-\varphi_{0}{ }^{*} \hat{g}\right)$ denotes the strain tensor field associated with the deformations $\varphi_{0}$ and $\varphi$.

The stress tensor field associated with a deformation $\varphi$ is the section

$$
\boldsymbol{\Sigma}[\varphi]:=\dddot{\boldsymbol{\Sigma}}\left(\cdot, E\left[\varphi_{0}, \varphi\right]\right) \in \mathscr{C}^{0}\left(S^{2} M \otimes \boldsymbol{\Lambda}^{n} M\right) .
$$

Its components $\Sigma[\varphi] \in \mathscr{C}^{0}\left(S^{2} M\right)$ and $\Sigma_{0}[\varphi] \in \mathscr{C}^{0}\left(S^{2} M\right)$ over the volume forms $\boldsymbol{\omega}[\varphi]$ and $\boldsymbol{\omega}_{0}$, which are defined by

$$
\boldsymbol{\Sigma}[\varphi]=\Sigma[\varphi] \otimes \boldsymbol{\omega}[\varphi]=\Sigma_{0}[\varphi] \otimes \boldsymbol{\omega}_{0},
$$

are also called stress tensor fields. Note that the tensor field $\Sigma[\varphi]$ is symmetric.

Other equivalent stress tensor fields are defined in terms of $\boldsymbol{\Sigma}[\varphi]$ by lowering and/or pushing forward some of its indices, viz.,

$$
\begin{array}{lll}
\boldsymbol{T}[\varphi]:=g[\varphi] \cdot \boldsymbol{\Sigma}[\varphi] & & \tilde{\boldsymbol{T}}[\varphi]:=(\hat{g} \circ \varphi) \cdot D \varphi \cdot \boldsymbol{\Sigma}[\varphi], \\
\hat{\boldsymbol{\Sigma}}[\varphi]:=\varphi_{*}(\Sigma[\varphi]) \otimes \hat{\boldsymbol{\omega}}, & & \hat{\boldsymbol{T}}[\varphi]:=\hat{g} \cdot \hat{\boldsymbol{\Sigma}}[\varphi],
\end{array}
$$

where - denotes the contraction of one index (no ambiguity should arise). The tensor fields $T[\varphi], T_{0}[\varphi] \in \mathscr{C}^{0}\left(T_{1}^{1} M\right)$ and $\tilde{T}[\varphi], \tilde{T}_{0}[\varphi] \in \mathscr{C}^{0}\left(T M \otimes \varphi^{*} T^{*} N\right)$ and $\hat{\Sigma}[\varphi] \in$ $\mathscr{C}^{0}\left(\left.S^{2} N\right|_{\varphi(M)}\right)$ and $\hat{T}[\varphi] \in \mathscr{C}^{0}\left(\left.T_{1}^{1} N\right|_{\varphi(M)}\right)$, defined by the decompositions

$$
\begin{array}{lll}
\boldsymbol{T}[\varphi]=T[\varphi] \otimes \boldsymbol{\omega}[\varphi]=T_{0}[\varphi] \otimes \boldsymbol{\omega}_{0}, & & \tilde{\boldsymbol{T}}[\varphi]=\tilde{T}[\varphi] \otimes \boldsymbol{\omega}[\varphi]=\tilde{T}_{0}[\varphi] \otimes \boldsymbol{\omega}_{0}, \\
\hat{\boldsymbol{\Sigma}}[\varphi]=\hat{\Sigma}[\varphi] \otimes \hat{\boldsymbol{\omega}}, & \hat{\boldsymbol{T}}[\varphi]=\hat{T}[\varphi] \otimes \hat{\boldsymbol{\omega}},
\end{array}
$$


are also called stress tensor fields.

Remark 3. When $(N, \hat{g})$ is the three-dimensional Euclidean space, the tensor fields $\tilde{T}_{0}[\varphi], \Sigma_{0}[\varphi]$, and $\hat{T}[\varphi]$, are respectively called the first Piola-Kirchhoff, the second Piola-Kirchhoff, and the Cauchy, stress tensor fields associated with the deformation $\varphi$.

The body and surface forces acting on the elastic body under consideration are assumed to be conservative.

The potential of these forces associated with a deformation $\varphi \in \operatorname{Def}(M, N)$ of the body is defined by

$$
P[\varphi]:=\int_{\varphi(M)} \hat{\boldsymbol{F}}[\varphi]+\int_{\varphi\left(\Gamma_{2}\right)} \hat{\boldsymbol{H}}[\varphi]=\int_{M} \varphi^{*}(\hat{\boldsymbol{F}}[\varphi])+\int_{\Gamma_{2}}\left(\left.\varphi\right|_{\Gamma_{2}}\right)^{*}(\hat{\boldsymbol{H}}[\varphi]),
$$

where $\hat{\boldsymbol{F}}[\varphi] \in \mathscr{C}^{0}\left(\boldsymbol{\Lambda}^{n} \varphi(M)\right)$ and $\hat{\boldsymbol{H}}[\varphi] \in \mathscr{C}^{0}\left(\boldsymbol{\Lambda}^{n-1} \varphi\left(\Gamma_{2}\right)\right), \Gamma_{2} \subset \Gamma:=\partial M$.

The work of the applied body and surface forces associated with a displacement field $\tilde{\xi}:=\hat{\xi} \circ \varphi$, where $\varphi \in \operatorname{Def}(M, N)$ and $\hat{\xi}:=\varphi_{*} \xi, \xi \in \mathscr{C}^{0}(T M)$, is the derivative $P^{\prime}[\varphi] \tilde{\xi}$. In what follows, we assume that the applied body and surface forces are local, i.e., that

$$
P^{\prime}[\varphi] \tilde{\xi}=\int_{\varphi(M)} \hat{\boldsymbol{f}}[\varphi] \cdot \hat{\xi}+\int_{\varphi\left(\Gamma_{2}\right)} \hat{\boldsymbol{h}}[\varphi] \cdot \hat{\xi}=\int_{M} \boldsymbol{f}[\varphi] \cdot \xi+\int_{\Gamma_{2}} \boldsymbol{h}[\varphi] \cdot \boldsymbol{\xi}
$$

where

$$
\begin{aligned}
\boldsymbol{f}[\varphi](x) & :=\dot{\boldsymbol{f}}(x, \varphi(x), D \varphi(x)), x \in M, \\
\boldsymbol{h}[\varphi](x) & =\dot{\boldsymbol{h}}(x, \varphi(x), D \varphi(x)), x \in \Gamma_{2} .
\end{aligned}
$$

The (given) mappings $\dot{\boldsymbol{f}}(x, y, \cdot)=\dot{f}_{0}(x, y, \cdot) \otimes \boldsymbol{\omega}_{0}(x): T_{x}^{*} M \otimes T_{y} N \rightarrow T^{*} M \otimes \boldsymbol{\Lambda}^{n} M$, $(x, y) \in M \times N$, and $\dot{\boldsymbol{h}}(x, y, \cdot)=\dot{h}_{0}(x, y, \cdot) \otimes \boldsymbol{\omega}_{0}(x):\left.T_{x}^{*} M \otimes T_{y} N \rightarrow T^{*} M\right|_{\Gamma_{2}} \otimes \boldsymbol{\Lambda}^{n} \Gamma_{2}$, $(x, y) \in \Gamma_{2} \times N$, are called the consitutice laws of the applied body and surface forces.

Remark 4. An example of such body and surface forces is obtained by assuming that the volume forms $\hat{\boldsymbol{F}}[\varphi]=\hat{\boldsymbol{F}}$ and $\hat{\boldsymbol{H}}[\varphi]=\hat{\boldsymbol{H}}$ are independent of the deformation $\varphi$. In this case,

$$
\boldsymbol{f}[\varphi] \cdot \xi=\varphi^{*}\left(\hat{\mathscr{L}}_{\varphi_{*} \xi} \hat{\boldsymbol{F}}\right) \text { and } \boldsymbol{h}[\varphi] \cdot \xi=\varphi^{*}\left(\hat{\mathscr{L}}_{\varphi_{*} \xi} \hat{\boldsymbol{H}}\right) \text { for all } \xi \in \mathscr{C}^{1}(T M)
$$

The densities of the applied body and surface forces are the sections

$$
\boldsymbol{f}[\varphi]=f[\varphi] \otimes \boldsymbol{\omega}[\varphi]=f_{0}[\varphi] \otimes \boldsymbol{\omega}_{0}, \quad \hat{\boldsymbol{f}}[\varphi]=\hat{f}[\varphi] \otimes \hat{\boldsymbol{\omega}}
$$

and

$$
\boldsymbol{h}[\varphi]=h[\varphi] \otimes \boldsymbol{i}_{v[\varphi]} \boldsymbol{\omega}[\varphi]=h_{0}[\varphi] \otimes \boldsymbol{i}_{v_{0}} \boldsymbol{\omega}_{0}, \quad \hat{\boldsymbol{h}}[\varphi]=\hat{h}[\varphi] \otimes \boldsymbol{i}_{\hat{v}[\varphi]} \hat{\boldsymbol{\omega}},
$$

where $v[\varphi], v_{0}$, and $\hat{v}[\varphi]$, denote the unit outer normal vector fields to the boundary of $(M, g[\varphi]),\left(M, g_{0}\right)$, and $(N, \hat{g})$, respectively. Note that $f[\varphi], f_{0}[\varphi], \hat{f}[\varphi], h[\varphi]$, $h_{0}[\varphi]$, and $\hat{h}[\varphi]$, are 1 -form fields. 


\section{The equations of nonlinear elasticity in a Riemannian manifold}

Let a reference deformation $\varphi_{0} \in \mathscr{C}^{3}(M, N)$ satisfy the assumptions of Section 4. Assume in addition that the body is kept fixed on a (possibly empty) portion $\varphi_{0}\left(\Gamma_{1}\right)$ of its boundary, where $\Gamma_{1} \subset \Gamma:=\partial M$ is a relatively open set, and is subjected to applied body and surface forces. Let $\Gamma_{2}:=\Gamma \backslash \Gamma_{1}$.

The principle of least energy asserts that the deformation $\varphi: M \rightarrow N$ of such a body should satisfy the following minimization problem:

$$
\varphi \in \Phi \text { and } J[\varphi] \leq J[\psi] \text { for all } \psi \in \Phi,
$$

where (see Section 3)

$$
\Phi:=\left\{\varphi \in \mathscr{C}^{1}(M, N) ;\left.\varphi\right|_{\text {int } M} \text { injective, } \operatorname{det} D \varphi>0 \text { in } M, \varphi=\varphi_{0} \text { on } \Gamma_{1}\right\}
$$

denotes the set of admissible deformations, and where (see Section 4)

$$
J[\varphi]:=I[\varphi]-P[\varphi]=\int_{M} \boldsymbol{W}[\varphi]-\left(\int_{M} \boldsymbol{F}[\varphi]+\int_{\Gamma_{2}} \boldsymbol{H}[\varphi]\right),
$$

denotes the total energy associated with the deformation $\varphi \in \Phi$. The mappings $\boldsymbol{W}, \boldsymbol{F}, \boldsymbol{H}: \Phi \rightarrow \boldsymbol{\Lambda}^{n} M$ are defined explicitely by the constitutive laws of the elastic materials and of the applied forces (see Section 4).

The next theorem identifies the variational equations, also called the principle of virtual work, that any solution to the minimization (23) should satisfy.

Theorem 2. A solution $\varphi \in \Phi$ to the minimization problem (23) satisfies the variational equations:

$$
\int_{M} \boldsymbol{\Sigma}[\varphi]: e[\varphi, \xi]=\int_{M} \boldsymbol{f}[\varphi] \cdot \xi+\int_{\Gamma_{2}} \boldsymbol{h}[\varphi] \cdot \xi
$$

for all $\xi \in \Xi:=\left\{\xi \in \mathscr{C}^{1}(T M) ; \xi=0\right.$ on $\left.\Gamma_{1}\right\}$.

Proof. Let $\varphi \in \mathscr{C}^{1}(M, N)$ be a solution to the minimization problem (23). Given any vector field $\xi \in \Xi$, let $\hat{\xi} \in \mathscr{C}^{1}(T N)$ denote any extension to $N$ of the vector field $\varphi_{*} \xi \in \mathscr{C}^{1}\left(\left.T N\right|_{\varphi(M)}\right)$. Let $\gamma_{\hat{\xi}}$ denote the flow of $\hat{\xi}$ (see Section 2) and define the time-dependent family of deformations

$$
\psi(\cdot, t):=\gamma_{\hat{\xi}}(\cdot, t) \circ \varphi, \quad t \in(-\varepsilon, \varepsilon) .
$$

Note that there exists $\varepsilon>0$ such that $\psi(\cdot, t) \in \Phi$ for all $t \in(-\varepsilon, \varepsilon)$.

Since $J[\varphi] \leq J[\psi(\cdot, t)]$ for all $t \in(-\varepsilon, \varepsilon)$, we deduce that $\left[\frac{d}{d t} J[\psi(\cdot, t)]\right]_{t=0}=0$, which implies in turn that

$$
\left[\frac{d}{d t} I[\psi(\cdot, t])\right]_{t=0}=\left[\frac{d}{d t} P[\psi(\cdot, t)]\right]_{t=0}=\int_{M} \boldsymbol{f}[\varphi] \cdot \xi+\int_{\Gamma_{2}} \boldsymbol{h}[\varphi] \cdot \xi .
$$


It remains to compute the first term of this relation.

Using the Lebesgue dominated convergence theorem, the chain rule, and the relations $\boldsymbol{W}[\varphi]=\dddot{\boldsymbol{W}}\left(\cdot, E\left[\varphi_{0}, \varphi\right]\right), \frac{\partial \dddot{\boldsymbol{W}}}{\partial E}(x, E)=\dddot{\boldsymbol{\Sigma}}(x, E), \boldsymbol{\Sigma}[\varphi]=\dddot{\boldsymbol{\Sigma}}\left(\cdot, E\left[\varphi_{0}, \varphi\right]\right)$, and $e[\varphi, \xi]=\left[\frac{d}{d t} E(\varphi, \psi(\cdot, t))\right]_{t=0}=\left[\frac{d}{d t} E\left(\varphi_{0}, \psi(\cdot, t)\right)\right]_{t=0}$, we deduce that

$$
\begin{aligned}
{\left[\frac{d}{d t} I[\psi(\cdot, t])\right]_{t=0} } & =\int_{M}\left[\frac{d}{d t} \dddot{\boldsymbol{W}}\left(\cdot, E\left[\varphi_{0}, \psi(\cdot, t)\right]\right)\right]_{t=0} \\
& =\int_{M} \dddot{\boldsymbol{\Sigma}}\left(\cdot, E\left[\varphi_{0}, \varphi\right]\right):\left[\frac{d}{d t} E\left(\varphi_{0}, \psi(\cdot, t)\right)\right]_{t=0} \\
& =\int_{M} \boldsymbol{\Sigma}[\varphi]: e[\varphi, \xi] .
\end{aligned}
$$

The next theorem identifies the boundary value problem that any sufficiently regular solution $\varphi$ of the variational equations of Theorem 2 should satisfy. The divergence operator appearing below corresponds to connection $\nabla=\nabla[\varphi]$; as such it depends on the unknown deformation $\varphi$ (see Section 2). The stress tensor field $\boldsymbol{T}[\varphi]=T[\varphi] \otimes \boldsymbol{\omega}[\varphi]$ is defined in Section 4.

Theorem 3. A deformation $\varphi \in \mathscr{C}^{2}(M, N)$ satisfies the variational equations of Theorem 2 if and only if

$$
\left\{\begin{array} { r l } 
{ - \operatorname { d i v } \boldsymbol { T } [ \varphi ] = \boldsymbol { f } [ \varphi ] } & { \text { in } \operatorname { i n t } M , } \\
{ \boldsymbol { T } [ \varphi ] _ { v } = \boldsymbol { h } [ \varphi ] } & { \text { on } \Gamma _ { 2 } , } \\
{ \varphi = \varphi _ { 0 } } & { \text { on } \Gamma _ { 1 } , }
\end{array} \Leftrightarrow \left\{\begin{array}{rl}
-\operatorname{div} T[\varphi]=f[\varphi] & \text { in } \operatorname{int} M, \\
T[\varphi] \cdot(v[\varphi] \cdot g[\varphi])=h[\varphi] & \text { on } \Gamma_{2}, \\
\varphi=\varphi_{0} & \text { on } \Gamma_{1},
\end{array}\right.\right.
$$

where $v:=v[\varphi]$ denotes the unit outer normal vector field to the boundary of $M$ corresponding to the metric tensor field $g[\varphi]:=\varphi^{*} \hat{g}$.

Proof. Let a deformation $\varphi \in \mathscr{C}^{2}(M, N)$ satisfy the variational equations of Theorem 2. Since $\boldsymbol{\Sigma}[\varphi]=\Sigma[\varphi] \otimes \boldsymbol{\omega}[\varphi]$ and $\Sigma[\varphi]$ is symmetric, we have, for each vector field $\xi \in \Xi$,

$$
\Sigma[\varphi]: e[\varphi, \xi]=\Sigma[\varphi]:(g[\varphi] \cdot \nabla \xi)=(\Sigma[\varphi] \cdot g[\varphi]): \nabla \xi=T[\varphi]: \nabla \xi .
$$

Therefore the variational equations of Theorem 2 are equivalent to

$$
\int_{M} \boldsymbol{T}[\varphi]: \nabla \xi=\int_{M} \boldsymbol{f}[\varphi] \cdot \xi+\int_{\Gamma_{2}} \boldsymbol{h}[\varphi] \cdot \xi \text { for all } \xi \in \Xi .
$$

The conclusion follows by applying the integration by parts formula of Lemma 1 to the integral appearing in the left-hand side.

We conclude this section by recasting the equations of nonlinear elasticity as variational equations, or as a boundary value problem, defined on the unknown deformed configuration $\varphi(M) \subset N$. By contrast to Theorems 2 and 3, the connection $\hat{\nabla}$ and the corresponding divergence operator $\widehat{\operatorname{div}}$ are fixed (independent 
of the unknown deformation $\varphi$ ). The stress tensor fields $\hat{\boldsymbol{\Sigma}}[\varphi]=\hat{\Sigma}[\varphi] \otimes \hat{\boldsymbol{\omega}}$ and $\hat{\boldsymbol{T}}[\varphi]=\hat{T}[\varphi] \otimes \hat{\boldsymbol{\omega}}$ are defined in Section 4.

Theorem 4. (a) A deformation $\varphi \in \mathscr{C}^{1}(M, N)$ satisfies the variational equations of Theorem 2 if and only if

$$
\int_{\varphi(M)} \hat{\boldsymbol{\Sigma}}[\varphi]: \hat{e}[\xi]=\int_{\varphi(M)} \hat{\boldsymbol{f}}[\varphi] \cdot \hat{\xi}+\int_{\varphi\left(\Gamma_{2}\right)} \hat{\boldsymbol{h}}[\varphi] \cdot \hat{\xi}
$$

for all $\hat{\xi}=\varphi_{*} \xi, \xi \in \Xi$.

(b) A deformation $\varphi \in \mathscr{C}^{2}(M, N)$ satisfies the variational equations (26) if and only if

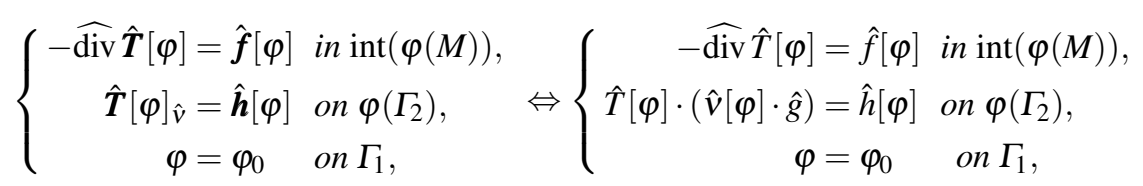

where $\hat{v}:=\hat{v}[\varphi]$ denotes the unit outer normal vector fields to the boundary of $\varphi(M)$ with respect to the metric tensor fields $\hat{g}$.

Proof. For each $\xi \in \Xi$ and $\hat{\xi}:=\varphi_{*} \xi$, we have

$$
\Sigma[\varphi]: e[\varphi, \xi]=\Sigma[\varphi]: \varphi^{*}(\hat{e}[\xi])=\left(\varphi_{*}(\Sigma[\varphi]): \hat{e}[\xi]\right) \circ \varphi=(\hat{\Sigma}[\varphi]: \hat{e}[\xi]) \circ \varphi ;
$$

hence

$$
\boldsymbol{\Sigma}[\varphi]: e[\varphi, \xi]=\varphi^{*}(\hat{\boldsymbol{\Sigma}}[\varphi]: \hat{e}[\hat{\xi}])
$$

Besides (see (21)),

$$
\boldsymbol{f}[\varphi] \cdot \xi=\varphi^{*}(\hat{\boldsymbol{f}}[\varphi] \cdot \hat{\xi}) \text { and } \boldsymbol{h}[\varphi] \cdot \xi=\varphi^{*}(\hat{\boldsymbol{h}}[\varphi] \cdot \hat{\xi}) .
$$

The last three relations and the change of variables formula show that the variational equations (26) are equivalent to those of Theorem 2.

Since $\hat{T}[\varphi]: \hat{\nabla} \hat{\xi}=\hat{\Sigma}[\varphi]: \hat{e}[\hat{\xi}]$, the variational equations (26) can be recast as

$$
\int_{\varphi(M)} \hat{\boldsymbol{T}}[\varphi]: \hat{\nabla} \hat{\xi}=\int_{\varphi(M)} \hat{\boldsymbol{f}}[\varphi] \cdot \hat{\xi}+\int_{\varphi\left(\Gamma_{2}\right)} \hat{\boldsymbol{h}}[\varphi] \cdot \hat{\xi} .
$$

Applying the integration by parts formula of Lemma 1 to the integral appearing in the left-hand side yields the announced boundary value problem.

\section{The equations of linearized elasticity in a Riemannian manifold}

The equations of linearized elasticity approach well the equations of nonlinear elasticity if the reference configuration $\varphi_{0}(M) \subset N, \varphi_{0} \in \mathscr{C}^{2}(M, N)$, of the elastic body 
under consideration is a natural state (that is, unconstrained) and if the applied forces are small enough.

The equations of linearized elasticity are deduced from the equations of nonlinear elasticity (see Theorems 2 and 3) by replacing the latter equations by their affine part with respect to the displacement field $\xi=\exp _{\varphi_{0}}^{-1} \varphi$. Thus the unknown in linearized elasticity is the vector field $\xi: M \rightarrow T M$, instead of the deformation $\varphi:=\exp _{\varphi_{0}} \xi$ : $M \rightarrow N$.

Let $\boldsymbol{\omega}_{0}, \boldsymbol{i}_{v_{0}} \boldsymbol{\omega}_{0}$, and $v_{0}$, respectively denote the volume form on $M$, the volume form on $\Gamma=\partial M$, and the unit outer normal vector field to the boundary of $M$, corresponding to the metric $g_{0}=g\left[\varphi_{0}\right]:=\varphi_{0}{ }^{*} \hat{g}$; see Section 2 .

Let $\boldsymbol{A}$ and $(\boldsymbol{f}[\varphi], \boldsymbol{h}[\varphi])$ respectively denote the elasticity tensor field and the densities of the applied forces appearing in the equations of nonlinear elasticity (see Theorems 2 and 3). For each vector field $\xi \in \mathscr{C}^{1}(T M)$, define

$$
\begin{array}{ll}
\boldsymbol{\Sigma}^{\operatorname{lin}}[\xi]:=\boldsymbol{A}: e\left[\varphi_{0}, \xi\right], & \boldsymbol{f}^{\mathrm{aff}}[\xi]:=\boldsymbol{f}\left[\varphi_{0}\right]+\boldsymbol{f}^{\prime}\left[\varphi_{0}\right] \xi, \\
\boldsymbol{T}^{\operatorname{lin}}[\xi]:=g_{0} \cdot \boldsymbol{\Sigma}^{\operatorname{lin}}[\xi], & \boldsymbol{h}^{\mathrm{aff}}[\xi]:=\boldsymbol{h}\left[\varphi_{0}\right]+\boldsymbol{h}^{\prime}\left[\varphi_{0}\right] \xi,
\end{array}
$$

where

$$
\begin{aligned}
& \boldsymbol{f}^{\prime}\left[\varphi_{0}\right] \xi:=\lim _{t \rightarrow 0} \frac{1}{t}\left(\boldsymbol{f}\left[\exp _{\varphi_{0}}(t \xi)\right]-\boldsymbol{f}\left[\varphi_{0}\right]\right)=\boldsymbol{f}^{1} \cdot \boldsymbol{\xi}+\boldsymbol{f}^{2}: \nabla_{0} \xi \\
& \boldsymbol{h}^{\prime}\left[\varphi_{0}\right] \xi:=\lim _{t \rightarrow 0} \frac{1}{t}\left(\boldsymbol{h}\left[\exp _{\varphi_{0}}(t \xi)\right]-\boldsymbol{h}\left[\varphi_{0}\right]\right)=\boldsymbol{h}^{1} \cdot \xi+\boldsymbol{h}^{2}: \nabla_{0} \xi
\end{aligned}
$$

for some sections $f^{1} \in \mathscr{C}^{0}\left(\boldsymbol{\Lambda}^{n} M \otimes T_{2}^{0} M\right), f^{2} \in \mathscr{C}^{0}\left(\boldsymbol{\Lambda}^{n} M \otimes T_{2}^{1} M\right), h^{1} \in \mathscr{C}^{0}\left(\boldsymbol{\Lambda}^{n-1} \Gamma_{2} \otimes\right.$ $\left.\left.T_{2}^{0} M\right|_{\Gamma_{2}}\right)$, and $\boldsymbol{h}^{2} \in \mathscr{C}^{0}\left(\left.\boldsymbol{\Lambda}^{n-1} \Gamma_{2} \otimes T_{2}^{1} M\right|_{\Gamma_{2}}\right)$. Then define the tensor fields $T_{0}^{\operatorname{lin}}[\xi] \in$ $\mathscr{C}^{1}\left(T_{1}^{1} M\right), f_{0}^{\text {aff }}[\xi] \in \mathscr{C}^{0}\left(T^{*} M\right)$ and $h_{0}^{\text {aff }}[\xi] \in \mathscr{C}^{0}\left(\left.T^{*} M\right|_{\Gamma_{2}}\right)$, by letting

$$
\begin{aligned}
\boldsymbol{T}^{\operatorname{lin}}[\xi] & =T_{0}^{\operatorname{lin}}[\xi] \otimes \boldsymbol{\omega}_{0}, \\
\boldsymbol{f}^{\mathrm{aff}}[\xi] & =f_{0}^{\mathrm{aff}}[\xi] \otimes \boldsymbol{\omega}_{0}, \\
\boldsymbol{h}^{\mathrm{aff}}[\xi] & =h_{0}^{\mathrm{aff}}[\xi] \otimes \boldsymbol{i}_{v_{0}} \boldsymbol{\omega}_{0} .
\end{aligned}
$$

We are now in a position to state the equations on linearized elasticity in a Riemannian manifold:

Theorem 5. (a) The vector field $\xi \in \mathscr{C}^{2}(T M)$ satisfies in linearized elasticity the following boundary value problem:

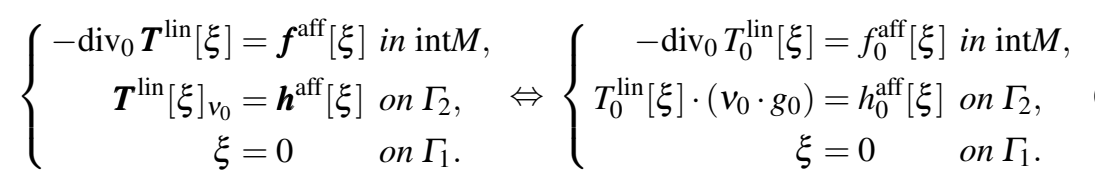

(b) The vector field $\xi \in \mathscr{C}^{1}(T M)$ satisfies in linearized elasticity the following variational equations: 


$$
\begin{aligned}
& \xi \in \Xi:=\left\{\eta \in \mathscr{C}^{1}(T M) ; \eta=0 \text { on } \Gamma_{1}\right\} \\
& \int_{M}\left(\boldsymbol{A}: e\left[\varphi_{0}, \xi\right]\right): e\left[\varphi_{0}, \eta\right]=\int_{M}\left(f^{\mathrm{aff}}[\xi]\right) \cdot \eta+\int_{\Gamma_{2}}\left(\boldsymbol{h}^{\mathrm{aff}}[\xi]\right) \cdot \eta \text { for all } \eta \in \Xi .
\end{aligned}
$$

Proof. (a) The boundary value problem of linearized elasticity is the affine (with respect to $\xi$ ) approximation of the following boundary value problem of nonlinear elasticity (see Theorem 3)

$$
\begin{aligned}
-\operatorname{div} \boldsymbol{T}[\varphi] & =\boldsymbol{f}[\varphi] & & \text { in } \operatorname{int} M, \\
\boldsymbol{T}[\varphi]_{v} & =\boldsymbol{h}[\varphi] & & \text { on } \Gamma_{2}, \\
\varphi & =\varphi_{0} & & \text { on } \Gamma_{1},
\end{aligned}
$$

satisfied by the deformation $\varphi:=\exp _{\varphi_{0}} \xi$. It remains to compute this affine approximation explicitly.

The dependence of the stress tensor field $\boldsymbol{T}[\varphi]$ on the vector field $\xi=\exp _{\varphi_{0}}^{-1} \varphi$ has been specified in Section 4 by means of the constitutive law of the elastic material, namely,

$$
\begin{aligned}
\boldsymbol{T}[\varphi](x) & =g[\varphi](x) \cdot \boldsymbol{\Sigma}[\varphi](x) \\
& =\left(\varphi^{*} \hat{g}\right)(x) \cdot \dddot{\boldsymbol{\Sigma}}\left(x, E\left[\varphi_{0}, \varphi\right](x)\right), x \in M .
\end{aligned}
$$

Since the reference configuration $\varphi_{0}(M)$ is a natural state, we have $\dddot{\Sigma}(x, 0)=0$ for all $x \in M$. The definition of the elasticity tensor field $\boldsymbol{A}$ next implies that

$$
\frac{\partial \dddot{\boldsymbol{\Sigma}}}{\partial E}(x, 0) H=\boldsymbol{A}(x): H \text { for all } x \in M \text { and all } H \in S_{2, x} M .
$$

Besides,

$$
\left[\frac{d}{d t} E\left[\varphi_{0}, \exp _{\varphi_{0}}(t \xi)\right]\right]_{t=0}=e\left[\varphi_{0}, \xi\right] \text { and }\left[\frac{d}{d t} g\left[\exp _{\varphi_{0}}(t \xi)\right]\right]_{t=0}=g_{0}
$$

The last three relations imply that

$$
\boldsymbol{T}[\varphi]=g[\varphi] \cdot \boldsymbol{\Sigma}[\varphi]=g_{0} \cdot\left(\boldsymbol{A}: e\left[\varphi_{0}, \xi\right]\right)+o\left(\|\xi\|_{\mathscr{C}^{1}(T M)}\right) .
$$

Since $\boldsymbol{T}^{\operatorname{lin}}[\xi]:=g_{0} \cdot\left(\boldsymbol{A}: e\left[\varphi_{0}, \xi\right]\right)$ is linear with respect to $\xi$, the previous relation implies that

$$
\operatorname{div} \boldsymbol{T}[\varphi]=\operatorname{div}_{0} \boldsymbol{T}^{\operatorname{lin}}[\xi]+o\left(\|\xi\|_{\mathscr{C}^{1}(T M)}\right),
$$

where div and $\operatorname{div}_{0}$ denote the divergence operators induced by the connections $\nabla:=\nabla[\varphi]$ and $\nabla_{0}:=\nabla\left[\varphi_{0}\right]$, respectively.

The dependence of the applied force densities $\boldsymbol{f}[\varphi]$ and $\boldsymbol{h}[\boldsymbol{\varphi}]$ on the vector field $\xi=\exp _{\varphi_{0}}^{-1} \varphi$ has been specified in Section 4 by means of the relations

$$
\boldsymbol{f}[\varphi](x)=\dot{\boldsymbol{f}}(x, \varphi(x), D \varphi(x)), x \in M, \text { and } \boldsymbol{h}[\varphi](x)=\dot{\boldsymbol{h}}(x, \varphi(x), D \varphi(x)), x \in \Gamma_{2} .
$$

Thus, using the notation (27) above, we have 


$$
\boldsymbol{f}[\varphi]=\boldsymbol{f}^{\mathrm{aff}}[\xi]+o\left(\|\xi\|_{\mathscr{C}^{1}(T M)}\right) \text { and } \boldsymbol{h}[\varphi]=\boldsymbol{h}^{\mathrm{aff}}[\xi]+o\left(\|\xi\|_{\mathscr{C}^{1}(T M)}\right)
$$

The boundary value problem (30) of linearized elasticity is then deduced from the boundary value problem (32) of nonlinear elasticity by using the estimates (33) and (34).

(b) The variational equations of linearized elasticity are the affine part with respect to $\xi$ of the variational equations of nonlinear elasticity (see Theorem 2)

$$
\mathscr{S}\left[\exp _{\varphi_{0}} \xi\right] \eta=0 \text { for all } \eta \in \Xi
$$

where

$$
\mathscr{S}[\varphi] \eta:=\int_{M} \boldsymbol{\Sigma}[\varphi]: e[\varphi, \eta]-\left(\int_{M} \boldsymbol{f}[\varphi] \cdot \eta+\int_{\Gamma_{2}} \boldsymbol{h}[\varphi] \cdot \eta\right)
$$

and $\boldsymbol{\Sigma}[\varphi]:=\dddot{\boldsymbol{\Sigma}}\left(\cdot, E\left[\varphi_{0}, \varphi\right]\right)$. Thus the variational equations of linearized elasticity satisfied by $\xi \in \Xi$ read:

$$
\mathscr{S}^{\operatorname{lin}}[\xi] \eta:=\mathscr{S}\left[\varphi_{0}\right] \eta+\left[\frac{d}{d t} \mathscr{S}\left[\exp _{\varphi_{0}}(t \xi)\right] \eta\right]_{t=0}=0 \text { for all } \eta \in \Xi .
$$

It remains to compute $\mathscr{S}^{\mathrm{lin}}[\xi] \eta$ explicitly. As in the proof of part (a),

$$
\boldsymbol{\Sigma}\left[\exp _{\varphi_{0}} \xi\right]=\boldsymbol{\Sigma}^{\operatorname{lin}}[\xi]+o\left(\|\xi\|_{\mathscr{C}^{1}(T M)}\right) .
$$

Besides, $\boldsymbol{\Sigma}^{\operatorname{lin}}[\xi]$ is linear, $e\left[\exp _{\varphi_{0}} \xi, \eta\right]=e\left[\varphi_{0}, \eta\right]+o\left(\|\xi\|_{\mathscr{C}^{1}(T M)}\right)$, and $\boldsymbol{f}[\varphi]$ and $\boldsymbol{h}[\varphi]$ satisfy relations (34). We then infer from the definition of $\mathscr{S}[\varphi] \eta$ that

$$
\mathscr{S}^{\operatorname{lin}}[\xi] \eta=\int_{M}\left(\boldsymbol{A}: e\left[\varphi_{0}, \xi\right]\right): e\left[\varphi_{0}, \eta\right]-\left(\int_{M}\left(\boldsymbol{f}^{\mathrm{aff}}[\xi]\right) \cdot \eta+\int_{\Gamma_{2}}\left(\boldsymbol{h}^{\mathrm{aff}}[\xi]\right) \cdot \eta\right)
$$

Remark 5. The variational equations (31) of linearized elasticity in a Riemannian manifold are extended by density to displacement fields $\xi \in H^{1}(T M)$ in order to prove that they possess solutions; cf. Theorem 7 .

\section{Existence and regularity theorem in linearized elasticity}

Throughout this section, the manifold $M$ is endowed with the Riemannian metric $g_{0}=g\left[\varphi_{0}\right]:=\varphi_{0}^{*} \hat{g}$, where $\varphi_{0}$ is a reference deformation of class $\mathscr{C}^{3}(M, N)$. As in the previous sections, $\nabla_{0}, \operatorname{div}_{0}$, and $\boldsymbol{\omega}_{0}$ denote the connection, the divergence operator, and the volume form on $M$ induced by $g_{0}$. The Sobolev spaces appearing below are defined in Section 2.

The existence of solutions to the equations of linearized elasticity in a Riemannian manifold relies on the following Riemannian version of Korn's inequality, due to Chen \& Jost [14]: 
Theorem 6. Assume that the differentiable manifold $M$ satisfies the following property: there exists a differentiable manifold $\tilde{M}$ of class $\mathscr{C}^{2}$ such that $M=\bar{\Omega} \subset \tilde{M}$, $\Omega$ is a bounded, connected, and open subset of $\tilde{M}$, and the boundary $\Gamma:=\partial M$ is non-empty and Lipschitz-continuous.

(a) There exists a constant $C_{1}$ depending on $\left(M, g_{0}\right)$ such that

$$
\|\xi\|_{H^{1}(T M)} \leq C_{1}\left(\|\xi\|_{L^{2}(T M)}+\left\|\mathscr{L}_{\xi} g_{0}\right\|_{L^{2}\left(S_{2} M\right)}\right)
$$

for all $\xi \in H^{1}(T M)$.

(b) Let $\Gamma_{1} \subset \Gamma$ be a non-empty relatively open subset of the boundary of $M$. There exists a constant $C_{K}$ depending on $\left(M, g_{0}\right)$ and on $\Gamma_{1}$ such that

$$
\|\xi\|_{H^{1}(T M)} \leq C_{K}\left\|\mathscr{L}_{\xi} g_{0}\right\|_{L^{2}\left(S_{2} M\right)}
$$

for all $\xi \in H^{1}(T M)$ satisfying $\xi=0$ on $\Gamma_{1}$.

Proof. We briefly sketch here for completeness the argument of Chen \& Jost [14], itself a generalization of the proof by Duvaut \& Lions [13] of the classical Korn inequality (classical means that $\left(M, g_{0}\right)$ is an Euclidean space).

Define the space

$$
X:=\left\{\xi \in L^{2}(T M) ; \mathscr{L}_{\xi} g_{0} \in L^{2}\left(S_{2} M\right)\right\}
$$

and endow it with the norm

$$
\|\xi\|_{X}:=\|\xi\|_{L^{2}(T M)}+\left\|\mathscr{L}_{\xi} g_{0}\right\|_{L^{2}\left(S_{2} M\right)} .
$$

Clearly, $H^{1}(T M) \subset X$. Let id : $H^{1}(T M) \rightarrow X$ denote the identity mapping. It suffices to prove that this mapping satisfies the assumptions of the open mapping theorem, since the continuity of the inverse mapping implies inequality (35).

The mapping id : $H^{1}(T M) \rightarrow X$ is injective and continuous, and the normed vector spaces $X$ and $H^{1}(T M)$ are both complete. It remains to prove that the mapping id : $H^{1}(T M) \rightarrow X$ is also surjective.

Let $\xi \in X$. $M$ being a compact subset of $\tilde{M}$, there exists a finite number of local charts $\tilde{\theta}_{\ell}: \tilde{V}_{\ell} \subset \tilde{M} \rightarrow \tilde{U}_{\ell} \subset \mathbb{R}^{n}, \ell \in\{1,2, \ldots, L\}$, of $\tilde{M}$ such that $M \subset \bigcup_{\ell=1}^{L} \tilde{V}_{\ell}$. Given any $\ell \in\{1,2, \ldots, L\}$, let $V:=\tilde{V}_{\ell} \cap M$, let $U:=\tilde{\theta}_{\ell}(V)$, and let $\theta:=\left.\tilde{\theta}_{\ell}\right|_{V}: V \subset M \rightarrow$ $U \subset \mathbb{R}^{n}$.

Let the functions $\xi_{i}: U \rightarrow \mathbb{R}$ and $e_{i j}: U \rightarrow \mathbb{R}$ denote the components in the local chart $\theta$ of $\xi^{b}:=g_{0} \cdot \xi$ and $e:=\frac{1}{2} \mathscr{L}_{\xi} g_{0}=\frac{1}{2}\left(\nabla_{0} \xi^{b}+\left(\nabla_{0} \xi^{b}\right)^{T}\right)$, respectively. Using Ricci's and Bianchi's identities (the notation below should be self-explanatory)

$$
\begin{aligned}
\nabla_{0 i}\left(\nabla_{0 j} \xi_{k}\right)-\nabla_{0 j}\left(\nabla_{0 i} \xi_{k}\right) & =-R_{k i j}^{\ell}\left(g_{0}\right) \xi_{\ell}, \\
R_{k i j}^{\ell}\left(g_{0}\right)+R_{i j k}^{\ell}\left(g_{0}\right)+R_{j k i}^{\ell}\left(g_{0}\right) & =0,
\end{aligned}
$$

and the anti-symmetry $R_{k i j}^{\ell}\left(g_{0}\right)=-R_{k j i}^{\ell}\left(g_{0}\right)$ of the components 


$$
R_{k i j}^{\ell}\left(g_{0}\right):=\frac{\partial \Gamma_{j k}^{\ell}\left(g_{0}\right)}{\partial x^{i}}-\frac{\partial \Gamma_{i k}^{\ell}\left(g_{0}\right)}{\partial x^{j}}+\Gamma_{i m}^{\ell}\left(g_{0}\right) \Gamma_{j k}^{m}\left(g_{0}\right)-\Gamma_{j m}^{\ell}\left(g_{0}\right) \Gamma_{i k}^{m}\left(g_{0}\right)
$$

of the Riemann curvature tensor field associated with the metric tensor field $g_{0}$, it is easy to see that

$$
\nabla_{0 i}\left(\nabla_{0 j} \xi_{k}\right)=\nabla_{0 i} e_{j k}+\nabla_{0 j} e_{k i}-\nabla_{0 k} e_{i j}+R_{i j k}^{\ell}\left(g_{0}\right) \xi_{\ell}
$$

Therefore, $\frac{\partial^{2} \xi_{k}}{\partial x^{i} \partial x^{j}} \in H^{-1}(U)$, which next implies that $\xi_{k} \in H^{1}(U)$ by a lemma due to J.L. Lions; see, e.g., [2] for domains $U$ with a Lipschitz-continuous boundary (as is the case here), or [13] for domains $U$ with smooth boundary. Hence $\xi \in H^{1}(T M)$, which proves that the mapping id: $H^{1}(T M) \rightarrow X$ is indeed surjective.

Inequality (36) is deduced from inequality (35) by a contradiction argument. So assume that inequality (36) were false for any constant $C_{K}$. Then, for each $\ell \in \mathbb{N}$, there exists $\xi_{\ell} \in H^{1}(T M)$ satisfying $\xi_{\ell}=0$ on $\Gamma_{1}$ such that $\left\|\xi_{\ell}\right\|_{H^{1}(T M)}>$ $\ell\left\|\mathscr{L}_{\xi_{\ell}} g_{0}\right\|_{L^{2}\left(S_{2} M\right)}$. Let $\eta_{\ell}:=\xi_{\ell} /\left\|\xi_{\ell}\right\|_{H^{1}(T M)}$. Then, for each $\ell \in \mathbb{N}$,

$$
\eta_{\ell} \in H^{1}(T M),\left.\quad \eta_{\ell}\right|_{\Gamma_{1}}=0, \quad\left\|\eta_{\ell}\right\|_{H^{1}(T M)}=1, \quad \text { and } \lim _{\ell \rightarrow \infty}\left\|\mathscr{L}_{\eta_{\ell} g_{0}}\right\|_{L^{2}\left(S_{2} M\right)}=0 .
$$

Since the space $H^{1}(T M)$ is reflexive, since the trace operator $\eta_{\ell} \in H^{1}(T M) \rightarrow$ $\left.\eta_{\ell}\right|_{\Gamma_{1}} \in L^{2}\left(\left.T M\right|_{\Gamma_{1}}\right)$ is linear and continuous, and since the embedding $H^{1}(T M) \subset$ $L^{2}(T M)$ is compact, there exists a subsequence, still indexed by $\ell$, of the sequence $\left(\eta_{\ell}\right)$ and an element $\eta \in H^{1}(T M),\left.\eta\right|_{\Gamma_{1}}=0$, such that

$$
\eta_{\ell} \rightarrow \eta \text { in } H^{1}(T M) \text { and } \eta_{\ell} \rightarrow \eta \text { in } L^{2}(T M), \quad \mathscr{L}_{\eta_{\ell}} g_{0} \rightarrow 0 \text { in } L^{2}\left(S_{2} M\right) .
$$

( $\rightarrow$ and $\rightarrow$ respectively denote weak and strong convergences). But

$$
\eta_{\ell} \rightarrow \eta \text { in } H^{1}(T M) \Rightarrow \mathscr{L}_{\eta_{\ell} g_{0}} \rightarrow \mathscr{L}_{\eta} g_{0} \text { in } L^{2}\left(S_{2} M\right) .
$$

Therefore, $\mathscr{L}_{\eta} g_{0}=0$ in $L^{2}\left(S_{2} M\right)$, which means that $\eta$ is a Killing vector field on $\left(M, g_{0}\right)$. Since in addition $\left.\eta\right|_{\Gamma_{1}}=0$, a property of Killing vector fields implies that $\eta=0$ in $M$; see, e.g., [14] or [20].

We just proved that

$$
\eta_{\ell} \rightarrow 0 \text { in } L^{2}(T M) \text { and } \mathscr{L}_{\eta_{\ell}} g_{0} \rightarrow 0 \text { in } L^{2}\left(S_{2} M\right) .
$$

By inequality (35), this next implies that

$$
\eta_{\ell} \rightarrow 0 \text { in } H^{1}(T M) \text {. }
$$

This contradicts that $\left\|\eta_{\ell}\right\|_{H^{1}(T M)}=1$ for all $\ell \in \mathbb{N}$.

The smallest possible constant $C_{K}$ for which Korn's inequality (36) holds is called the Korn constant of $\left(M, g_{0}\right)$ and $\Gamma_{1} \subset \partial M$. It plays an important role in both linearized elasticity and nonlinear elasticity (see assumptions (38) and (55) of Theorems 7 and 8, respectively) since the smaller the Korn constant is, the larger the 
applied forces are in both existence theorems. To our knowledge, the dependence of the Korn constant on the metric $g_{0}$ of $M$ and on $\Gamma_{1}$ is currently unknown, save a few particular cases; see, e.g., [18].

One such particular case, relevant to Theorem 8 , is when $\Gamma_{1}=\partial M$ and the metric $g_{0}$ is close to a flat metric, in the sense that its Ricci tensor field $\operatorname{Ric}_{0}:=\operatorname{Ricci}\left(g_{0}\right)$ satisfies the inequality $\left\|\operatorname{Ric}_{0}\right\|_{L^{\infty}\left(S_{2} M\right)} \leq \frac{1}{C_{P}}$, where $C_{P}$ is a Poincaré constant of $\left(M, g_{0}\right)$, i.e., a constant that satisfies

$$
\|\xi\|_{L^{2}(T M)}^{2} \leq C_{P}\left\|\nabla_{0} \xi\right\|_{L^{2}\left(T_{1}^{1} M\right)}^{2} \text { for all } \xi \in H_{0}^{1}(T M) .
$$

To see this, it suffices to combine the inequality

$$
\begin{array}{r}
\left\|\nabla_{0} \xi\right\|_{L^{2}\left(T_{1}^{1} M\right)}^{2}+\left\|\operatorname{div}_{0} \xi\right\|_{L^{2}(M)}^{2}=\frac{1}{2}\left\|\mathscr{L}_{\xi} g_{0}\right\|_{L^{2}\left(S_{2} M\right)}^{2}+\int_{M} \operatorname{Ric}_{0}(\xi, \xi) \boldsymbol{\omega}_{0} \\
\leq \frac{1}{2}\left\|\mathscr{L}_{\xi} g_{0}\right\|_{L^{2}\left(S_{2} M\right)}^{2}+\left\|\operatorname{Ric}_{0}\right\|_{L^{\infty}\left(S_{2} M\right)}\|\xi\|_{L^{2}(T M)}^{2}
\end{array}
$$

which holds for all $\xi \in H_{0}^{1}(T M)$, with the above assumption on the Ricci tensor field of $g_{0}$ to deduce that

$$
\left\|\nabla_{0} \xi\right\|_{L^{2}\left(T_{1}^{1} M\right)}^{2} \leq \frac{1}{2\left(1-C_{P}\left\|\operatorname{Ric}_{0}\right\|_{L^{\infty}\left(S_{2} M\right)}\right)}\left\|\mathscr{L}_{\xi} g_{0}\right\|_{L^{2}\left(S_{2} M\right)}^{2} .
$$

Hence the constant $C_{K}=\left\{2\left(1-C_{P}\left\|\operatorname{Ric}_{0}\right\|_{L^{\infty}\left(S_{2} M\right)}\right)\right\}^{-1}$ can be used in Theorems 7 and 8 when $\Gamma_{1}=\partial M$ and $\left\|\operatorname{Ric}_{0}\right\|_{L^{\infty}\left(S_{2} M\right)} \leq \frac{1}{C_{P}}$. Interestingly enough, particularizing these theorems to a flat metric $g_{0}$ yields existence theorems in classical elasticity with $C_{K}=1 / 2$, which is optimal.

The next theorem establishes the existence and regularity of the solution to the equations of linearized elasticity under specific assumptions on the data. Recall that the applied body and surface forces in linearized elasticity are of the form

$$
\begin{aligned}
& \boldsymbol{f}^{\mathrm{aff}}[\xi]=\boldsymbol{f}\left[\varphi_{0}\right]+\boldsymbol{f}^{\prime}\left[\varphi_{0}\right] \xi=\boldsymbol{f}\left[\varphi_{0}\right]+\left(\boldsymbol{f}^{1} \cdot \xi+\boldsymbol{f}^{2}: \nabla_{0} \xi\right), \\
& \boldsymbol{h}^{\mathrm{aff}}[\xi]=\boldsymbol{h}\left[\varphi_{0}\right]+\boldsymbol{h}^{\prime}\left[\varphi_{0}\right] \xi=\boldsymbol{h}\left[\varphi_{0}\right]+\left(\boldsymbol{h}^{1} \cdot \xi+\boldsymbol{h}^{2}: \nabla_{0} \xi\right),
\end{aligned}
$$

cf. relations (27) -(28). We say that the elasticity tensor field $\boldsymbol{A}=A_{0} \otimes \boldsymbol{\omega}_{0}$ of an elastic material is uniformly positive-definite if there exists a constant $C_{A_{0}}>0$ such that

$$
\left(A_{0}(x): H(x)\right): H(x) \geq C_{A_{0}}|H(x)|^{2} \text { for almost all } x \in M \text { and all } H(x) \in S_{2, x} M,
$$

where

$$
|H(x)|^{2}:=g_{0}(x)(H(x), H(x))
$$

and, in any local chart,

$$
\left(A_{0}(x): H(x)\right): H(x) \stackrel{\text { def }}{=} A_{0} i j k \ell(x) H_{k \ell}(x) H_{i j}(x) .
$$


Theorem 7. Let the Riemannian manifold $\left(M, g_{0}\right)$ satisfy the assumptions of Theorem 6. Assume that $\Gamma_{1} \subset \partial M$ is a non-empty relatively open subset of the boundary of $M$, that the elasticity tensor field $\boldsymbol{A}=A_{0} \otimes \boldsymbol{\omega}_{0}$ is essentially bounded and uniformly positive-definite, and that the applied body and surface forces satisfy the smallness assumption

$$
\left.\| \boldsymbol{f}^{\prime}\left[\varphi_{0}\right]\right]\left\|_{\mathscr{L}\left(H^{1}(T M), L^{2}\left(T^{*} M \otimes \boldsymbol{\Lambda}^{n} M\right)\right)}+\right\| \boldsymbol{h}^{\prime}\left[\boldsymbol{\varphi}_{0}\right] \|_{\mathscr{L}\left(H^{1}(T M) L^{2}\left(\left.T^{*} M\right|_{\Gamma_{2}} \otimes \boldsymbol{\Lambda}^{n-1} \Gamma_{2}\right)\right)} \leq \frac{C_{A_{0}}}{C_{K}},
$$

where $C_{K}$ denotes the constant appearing in Korn's inequality (36).

(a) If $\boldsymbol{f}\left[\varphi_{0}\right] \in L^{2}\left(T^{*} M \otimes \boldsymbol{\Lambda}^{n} M\right)$ and $\boldsymbol{h}\left[\varphi_{0}\right] \in L^{2}\left(\left.T^{*} M\right|_{\Gamma_{2}} \otimes \boldsymbol{\Lambda}^{n-1} \Gamma_{2}\right)$, there exists a unique vector field $\xi \in H^{1}(T M), \xi=0$ on $\Gamma_{1}$, such that

$$
\int_{M}\left(A: e\left[\varphi_{0}, \xi\right]\right): e\left[\varphi_{0}, \eta\right]=\int_{M} f^{\mathrm{aff}}[\xi] \cdot \eta+\int_{\Gamma_{2}} h^{\mathrm{aff}}[\xi] \cdot \eta
$$

for all $\eta \in H^{1}(T M), \eta=0$ on $\Gamma_{1}$.

(b) Assume in addition that $\Gamma_{1}=\partial M$ and, for some $m \in \mathbb{N}$ and $1<p<\infty$, the boundary of $M$ is of class $\mathscr{C}^{m+2}, \varphi_{0} \in \mathscr{C}^{m+2}(M, N), \boldsymbol{A} \in \mathscr{C}^{m+1}\left(T_{0}^{4} M \otimes \boldsymbol{\Lambda}^{n} M\right), \boldsymbol{f}^{1} \in$ $\mathscr{C}^{m}\left(T_{2}^{0} M \otimes \boldsymbol{\Lambda}^{n} M\right), \boldsymbol{f}^{2} \in \mathscr{C}^{m}\left(T_{2}^{1} M \otimes \boldsymbol{\Lambda}^{n} M\right)$, and $\boldsymbol{f}\left[\varphi_{0}\right] \in W^{m, p}\left(T^{*} M \otimes \boldsymbol{\Lambda}^{n} M\right)$. Then $\xi \in W^{m+2, p}(T M)$ and satisfies the boundary value problem

$$
\begin{aligned}
-\operatorname{div}_{0}\left(\boldsymbol{T}^{\operatorname{lin}}[\xi]\right) & =f^{\text {aff }}[\xi] & & \text { in } M, \\
\xi & =0 & & \text { on } \partial M .
\end{aligned}
$$

Furthermore, the mapping $\mathscr{A}^{\text {lin }}: W^{m+2, p}(T M) \rightarrow W^{m, p}\left(T^{*} M \otimes \boldsymbol{\Lambda}^{n} M\right)$ defined by

$$
\mathscr{A}^{\operatorname{lin}}[\eta]:=\operatorname{div}_{0} \boldsymbol{T}^{\operatorname{lin}}[\eta]+\boldsymbol{f}^{\prime}\left[\varphi_{0}\right] \eta \quad \text { for all } \eta \in W^{m+2, p}(T M),
$$

is linear, bijective, continuous, and its inverse $\left(\mathscr{A}^{\text {lin }}\right)^{-1}$ is also linear and continuous.

Proof. (a) Korn's inequality, the uniform positive-definiteness of $\boldsymbol{A}$, and the smallness of the linear part of the applied forces (see (36), (37), and (38)), together imply by means of Lax-Milgram theorem that the variational equations of linearized elasticity (39) possess a unique solution $\xi$ in the space $\left\{\xi \in H^{1}(T M)\right.$; $\xi=0$ on $\left.\Gamma_{1}\right\}$.

(b) It is clear that the solution of (39) is a weak solution to the boundary value problem (40). Since the latter is locally (in any local chart) an elliptic system of linear partial differential equations, the regularity assumptions on $\boldsymbol{A}$ and $\boldsymbol{f}^{\text {aff }}$ and the standard theory of elliptic systems of partial differential equations imply that this solution is locally of class $W^{m+2, p}$; see, e.g., the proof of Theorem 6.3-6 in [10]. Furthermore, the regularity of the boundary of $M$ together with the assumption that $\Gamma_{1}=\partial M$ imply that $\xi \in W^{m+2, p}(T M)$.

The mapping $\mathscr{A}^{\text {lin }}$ defined in the theorem is clearly linear and continuous. It is injective, since $\mathscr{A}^{\operatorname{lin}}[\xi]=0$ with $\xi \in W^{m+2, p}(T M)$ implies that $\xi$ satisfies the variational equations (39), hence $\xi=0$ by the uniqueness part of (a). It is also surjective since, given any $f_{0} \in W^{m, p}\left(T^{*} M \otimes \boldsymbol{\Lambda}^{n} M\right)$, there exists $\xi \in H_{0}^{1}(T M)$ such 
that $\int_{M}\left(\boldsymbol{A}: e\left[\varphi_{0}, \xi\right]\right): e\left[\varphi_{0}, \eta\right]=\int_{M} f_{0} \cdot \eta$ for all $\eta \in H_{0}^{1}(T M)$ (by part (a) of the theorem), and $\xi \in W^{m+2, p}(T M)$ by the regularity result established above. That the inverse of $\mathscr{A}^{\text {lin }}$ is also linear and continuous follows from the open mapping theorem.

Remark 6. The regularity assumption $\boldsymbol{A} \in \mathscr{C}^{m+1}\left(T_{0}^{4} M \otimes \boldsymbol{\Lambda}^{n} M\right)$ can be replaced in Theorem 7(b) by the weaker regularity $\boldsymbol{A} \in W^{m+1, p}\left(T_{0}^{4} M \otimes \boldsymbol{\Lambda}^{n} M\right),(m+1) p>n:=$ $\operatorname{dim} M$, by using improved regularity theorems for elliptic systems of partial differential equations; cf. [25].

\section{Existence theorem in nonlinear elasticity}

We show in this section that the boundary value problem of nonlinear elasticity in a Riemannian manifold (see Theorem 3) possesses at least a solution in an appropriate Sobolev space if $\Gamma_{2}=\emptyset$ and if the applied body forces are sufficiently small in a sense specified below. The assumption that $\Gamma_{2}=\emptyset$ means that the boundary value problem is of pure Dirichlet type, that is, the boundary condition $\varphi=\varphi_{0}$ is imposed on the whole boundary $\Gamma_{1}=\Gamma$ of the manifold $M$. Thus our objective is to prove the existence of a deformation $\varphi: M \rightarrow N$ that satisfies the system (see Theorem (3)):

$$
\begin{aligned}
-\operatorname{div} \boldsymbol{T}[\varphi] & =\boldsymbol{f}[\varphi] & & \text { in } \operatorname{int} M, \\
\varphi & =\varphi_{0} & & \text { on } \Gamma,
\end{aligned}
$$

where

$$
\begin{aligned}
\boldsymbol{T}[\varphi](x) & :=g[\varphi](x) \cdot \dddot{\boldsymbol{\Sigma}}\left(x, E\left[\varphi_{0}, \varphi\right](x)\right), x \in M, \\
\boldsymbol{f}[\varphi](x) & :=\dot{\boldsymbol{f}}(x, \varphi(x), D \varphi(x)), x \in M,
\end{aligned}
$$

the functions $\dddot{\boldsymbol{\Sigma}}$ and $\dot{\boldsymbol{f}}$ being the constitutive laws of the elastic material and of the applied forces, respectively (see Section 4). Recall that the divergence operator $\operatorname{div}=\operatorname{div}[\varphi]$ depends itself on the unknown $\varphi$ (since it is induced by the metric $\left.g=g[\varphi]:=\varphi^{*} \hat{g}\right)$ and that $\boldsymbol{\omega}=\boldsymbol{\omega}[\varphi]:=\varphi^{*} \hat{\boldsymbol{\omega}}$ denotes the volume form on $M$ corresponding to the metric $g[\varphi]$.

The idea is to seek a solution of the form $\varphi:=\exp _{\varphi_{0}} \xi$, where $\varphi_{0}: M \rightarrow N$ denotes a natural configuration of the body and $\xi: M \rightarrow T M$ is a sufficiently regular vector field in the set

$$
\mathscr{C}_{\varphi_{0}}^{0}(T M):=\left\{\xi \in \mathscr{C}^{0}(T M) ;\left\|\varphi_{0_{*}} \xi\right\|_{\mathscr{C}^{0}\left(\left.T N\right|_{\varphi(M)}\right)}<\hat{\delta}\left(\varphi_{0}(M)\right)\right\},
$$

where $\hat{\delta}\left(\varphi_{0}(M)\right)$ denotes the injectivity radius of the compact subset $\varphi_{0}(M)$ of $N$; see (7) in Section 3. It is then clear that the deformation $\varphi:=\exp _{\varphi_{0}} \xi, \xi \in \mathscr{C}^{1}(T M) \cap$ $\mathscr{C}_{\varphi_{0}}^{0}(T M)$, satisfies the boundary value problem (42) if and only if the displacement field $\xi$ satisfies the boundary value problem (the divergence operator below depends on the unknown $\xi$ ) 


$$
\begin{aligned}
-\operatorname{div} \boldsymbol{T}\left[\exp _{\varphi_{0}} \xi\right] & =\boldsymbol{f}\left[\exp _{\varphi_{0}} \xi\right] & & \text { in } \operatorname{int} M, \\
\xi & =0 & & \text { on } \Gamma .
\end{aligned}
$$

Given any vector field $\xi \in \mathscr{C}^{1}(T M) \cap \mathscr{C}_{\varphi_{0}}^{0}(T M)$, let

$$
\mathscr{A}[\xi]:=\operatorname{div}\left(\boldsymbol{T}\left[\exp _{\varphi_{0}} \xi\right]\right)+\boldsymbol{f}\left[\exp _{\varphi_{0}} \xi\right] .
$$

Proving an existence theorem to the boundary value problem (44) amounts to proving the existence of a solution to the equation $\mathscr{A}[\xi]=0$ in an appropriate space of vector fields $\xi: M \rightarrow T M$ satisfying the boundary condition $\xi=0$ on $\Gamma$. This will be done by using a variant of Newton's method, which seeks a zero of $\mathscr{A}$ as the limit of the sequence defined by

$$
\xi_{1}:=0 \text { and } \xi_{k+1}:=\xi_{k}-\mathscr{A}^{\prime}[0]^{-1} \mathscr{A}\left[\xi_{k}\right], k \geq 1 .
$$

Another way to prove the existence of a zero of $\mathscr{A}$ is to apply the NewtonKantorovich theorem (see, e.g., [12]), or the local inversion theorem (see Remark 7(a) below), to the mapping $\mathscr{A}$, but the result would be weaker than Theorem 8 below.

The key to applying Newton's method is to find function spaces $X$ and $\boldsymbol{Y}$ such that the mapping $\mathscr{A}: U \subset X \rightarrow \boldsymbol{Y}$ be differentiable in a neighborhood $U$ of $\xi=0 \in X$. The definition (45) of $\mathscr{A}$ can be recast in the equivalent form

$$
\mathscr{A}[\xi]:=\operatorname{div}\left(\left(\boldsymbol{T} \circ \exp _{\varphi_{0}}\right)[\xi]\right)+\left(\boldsymbol{f} \circ \exp _{\varphi_{0}}\right)[\xi],
$$

where the mappings $\left(\boldsymbol{T} \circ \exp _{\varphi_{0}}\right)$ and $\left(\boldsymbol{f} \circ \exp _{\varphi_{0}}\right)$ are defined at each $x \in M$ by the constitutive equations (the mappings $\dddot{\boldsymbol{\Sigma}}$ and $\dot{\boldsymbol{f}}$ are those appearing in (43))

$$
\begin{aligned}
\left(\left(\boldsymbol{T} \circ \exp _{\varphi_{0}}\right)[\xi]\right)(x) & =\dddot{\boldsymbol{T}}\left(x, \xi(x), \nabla_{0} \xi(x)\right):=g[\varphi](x) \cdot \dddot{\boldsymbol{\Sigma}}\left(x, E\left[\varphi_{0}, \varphi\right](x)\right), \\
\left(\left(\boldsymbol{f} \circ \exp _{\varphi_{0}}\right)[\xi]\right)(x) & =\dddot{\boldsymbol{f}}\left(x, \xi(x), \nabla_{0} \xi(x)\right):=\dot{\boldsymbol{f}}(x, \varphi(x), D \varphi(x)),
\end{aligned}
$$

where $\varphi=\exp _{\varphi_{0}} \xi$, for all vector fields $\xi \in \mathscr{C}^{1}(T M) \cap \mathscr{C}_{\varphi_{0}}^{0}(T M)$.

Relations (48) show that $\left(\boldsymbol{T} \circ \exp _{\varphi_{0}}\right)$ and $\left(\boldsymbol{f} \circ \exp _{\varphi_{0}}\right)$ are Nemytskii (or substitution) operators. It is well known that such operators are not differentiable between Lebesgue spaces unless they are linear, essentially because these spaces are not stable under multiplication. Therefore $\xi$ must belong to a space $X$ with sufficient regularity, so that the nonlinearity of $\dddot{\boldsymbol{T}}$ and $\dddot{\boldsymbol{f}}$ with respect to $\left(\xi(x), \nabla_{0} \xi(x)\right)$ be compatible with the desired differentiability of $\mathscr{A}$. Since we also want $\xi$ to belong to a reflexive Sobolev space (so that we could use the theory of elliptic systems of partial differential equations), we set

$$
X:=W^{m+2, p}(T M) \cap W_{0}^{1, p}(T M) \text { and } \boldsymbol{Y}:=W^{m, p}\left(T^{*} M \otimes \boldsymbol{\Lambda}^{n} M\right)
$$

for some $m \in \mathbb{N}$ and $1<p<\infty$ satisfying $(m+1) p>n$, and endow these space with the norms $\|\cdot\|_{X}:=\|\cdot\|_{m+2, p}$ and $\|\cdot\|_{\boldsymbol{Y}}:=\|\cdot\|_{m, p}$, respectively. 
Note that the normed spaces $X$ and $\boldsymbol{Y}$ are complete, and that the condition $(m+$ 1) $p>n$ is needed to ensure that the Sobolev space $W^{m+1, p}\left(T_{1}^{1} M\right)$, to which $\nabla_{0} \xi$ belongs, is stable under multiplication. It also implies that $X \subset \mathscr{C}^{1}(T M)$, so the deformation $\varphi=\exp _{\varphi_{0}} \xi$ induced by a vector field $\xi \in X \cap \mathscr{C}_{\varphi_{0}}^{0}(T M)$ is at least of class $\mathscr{C}^{1}$; hence the nonlinear model of elasticity make sense for $\xi \in X \cap \mathscr{C}_{\varphi_{0}}^{0}(T M)$.

Define

$$
U=B_{X}(\delta):=\left\{\xi \in X ;\|\xi\|_{X}<\delta\right\} \subset X
$$

as an open ball in $X$ centered at the origin over which the exponential map $\varphi=$ $\exp _{\varphi_{0}} \xi$ is well-defined. It suffices for instance to set

$$
\delta=\delta\left(\varphi_{0}, m, p\right):=\frac{\hat{\delta}\left(\varphi_{0}(M)\right)}{C_{S}(m+2, p)\left\|D \varphi_{0}\right\|_{\mathscr{C}^{0}\left(T^{*} M \otimes \varphi_{0}^{*} T N\right)}},
$$

where $C_{S}(m+2, p)$ denotes the norm of the Sobolev embedding $W^{m+2, p}(T M) \subset$ $\mathscr{C}^{0}(T M)$, since, for all $\xi \in B_{X}(\delta)$,

$$
\begin{aligned}
\left\|\varphi_{0 *} \xi\right\|_{\mathscr{C}^{0}\left(\left.T N\right|_{\varphi_{0}(M)}\right)} & =\sup _{x \in M}\left|D \varphi_{0}(x) \cdot \xi(x)\right| \\
& \leq\left\|D \varphi_{0}\right\|_{\mathscr{C}^{0}\left(T^{*} M \otimes \varphi_{0}^{*} T N\right)} C_{S}(m+2, p)\|\xi\|_{X} \\
& <\hat{\delta}\left(\varphi_{0}(M)\right) .
\end{aligned}
$$

We assume that the reference configuration $\varphi_{0}(M) \subset N$ of the elastic body under consideration is a natural state, and that the reference deformation, the constitutive law of the elastic material constituting the body, and the applied body forces defined by (48), satisfy the following regularity assumptions:

$$
\begin{aligned}
\varphi_{0} & \in \mathscr{C}^{m+2}(M, N), \\
\dddot{\boldsymbol{T}} & \in \mathscr{C}^{m+1}\left(M \times T M \times T_{1}^{1} M, T_{1}^{1} M \otimes \boldsymbol{\Lambda}^{n} M\right), \\
\left(\dddot{\boldsymbol{f}}-\boldsymbol{f}\left[\varphi_{0}\right]\right) & \in \mathscr{C}^{m}\left(M \times T M \times T_{1}^{1} M, T^{*} M \otimes \boldsymbol{\Lambda}^{n} M\right),
\end{aligned}
$$

and

$$
\boldsymbol{f}\left[\varphi_{0}\right] \in W^{m, p}\left(T^{*} M \otimes \boldsymbol{\Lambda}^{n} M\right),
$$

for some $m \in \mathbb{N}$ and $p \in(1, \infty)$ satisfying $(m+1) p>n$. Under these assumptions, standard arguments about composite mappings and the fact that $W^{m+1, p}(M)$ is an algebra together imply that the mappings

$$
\begin{gathered}
\left(\boldsymbol{T} \circ \exp _{\varphi_{0}}\right): \xi \in B_{X}(\boldsymbol{\delta}) \rightarrow \boldsymbol{T}\left[\exp _{\varphi_{0}} \xi\right] \in W^{m+1, p}\left(T_{1}^{1} \boldsymbol{M} \otimes \boldsymbol{\Lambda}^{n} M\right), \\
\left(\boldsymbol{f} \circ \exp _{\varphi_{0}}\right): \xi \in B_{X}(\boldsymbol{\delta}) \rightarrow \boldsymbol{f}\left[\exp _{\varphi_{0}} \xi\right] \in W^{m, p}\left(T^{*} \boldsymbol{M} \otimes \boldsymbol{\Lambda}^{n} M\right),
\end{gathered}
$$

are of class $\mathscr{C}^{1}$ over the open subset $B_{X}(\delta)$ of the Banach space $X$. Since $\mathscr{A}[\xi]=$ $\operatorname{div} \boldsymbol{T}\left[\exp _{\varphi_{0}} \xi\right]+\boldsymbol{f}\left[\exp _{\varphi_{0}} \xi\right]$ for all $\xi \in B_{X}(\delta)$, the mapping $\mathscr{A}$ belongs to the space $\mathscr{C}^{1}\left(B_{X}(\boldsymbol{\delta}), \boldsymbol{Y}\right)$. 
Finally, we assume that the elasticity tensor field $\boldsymbol{A}=A_{0} \times \boldsymbol{\omega}_{0}$, where $\boldsymbol{\omega}_{0}:=$ $\varphi_{0}^{*} \hat{\boldsymbol{\omega}}$, of the elastic material constituting the body under consideration is uniformly positive-definite, that is, there exists a constant $C_{A_{0}}>0$ such that

$$
\left(A_{0}(x): H(x)\right): H(x) \geq C_{A_{0}}|H(x)|^{2}, \text { where }|H(x)|^{2}:=g_{0}(x)(H(x), H(x)),
$$

for almost all $x \in M$ and all $H(x) \in S_{2, x} M$ (the same condition as in linearized elasticity; see (37)).

We are now in a position to establish the existence of a solution to the Dirichlet boundary value problem of nonlinear elasticity in a Riemannian manifold if the density $f\left[\varphi_{0}\right]$, resp. the first variation $f^{\prime}\left[\varphi_{0}\right]$, of the applied body forces acting on, resp. in a neighborhood of, the reference configuration $\varphi_{0}(M)$ are both small enough in appropriate norms.

Theorem 8. Suppose that the reference deformation $\varphi_{0}$ and the constitutive laws $\dddot{T}$ and $\dddot{f}$ satisfy the regularity assumptions (52) and (53), that the elasticity tensor field $\boldsymbol{A}=A_{0} \otimes \boldsymbol{\omega}_{0}$ satisfy the inequality (54), and that the manifold $M$ possesses a non-empty boundary of class $\mathscr{C}^{m+2}$. Let $\mathscr{A}: B_{X}(\delta) \subset X \rightarrow \boldsymbol{Y}$ denote the (possibly nonlinear) mapping defined by (45), (49), and (51).

(a) Assume that the first variation of the density of the applied body forces at $\varphi_{0}$ satisfies the smallness assumption:

$$
\left\|\boldsymbol{f}^{\prime}\left[\varphi_{0}\right]\right\|_{\mathscr{L}\left(H^{1}(T M), L^{2}\left(T^{*} M \otimes \boldsymbol{\Lambda}^{n} M\right)\right)} \leq C_{A_{0}} / C_{K},
$$

where $C_{K}$ denotes the constant appearing in Korn's inequality (36).

Then the mapping $\mathscr{A}$ is differentiable over the open ball $B_{X}(\delta)$ of $X, \mathscr{A}^{\prime}[0] \in$ $\mathscr{L}(X, \boldsymbol{Y})$ is bijective, and $\mathscr{A}^{\prime}[0]^{-1} \in \mathscr{L}(\boldsymbol{Y}, X)$. Moreover, $\mathscr{A}^{\prime}[0]=\mathscr{A}^{\text {lin }}$ is precisely the differential operator of linearized elasticity defined by (41).

(b) Assume in addition that the density of the applied body forces acting on the reference configuration $\varphi_{0}(M)$ of the body satisfies the smallness assumption:

$$
\left\|\boldsymbol{f}\left[\varphi_{0}\right]\right\|_{\boldsymbol{Y}}<\varepsilon_{1}:=\sup _{0<r<\delta} r\left(\left\|\mathscr{A}^{\prime}[0]^{-1}\right\|_{\mathscr{L}(\boldsymbol{Y}, X)}^{-1}-\sup _{\|\xi\|_{X}<r}\left\|\mathscr{A}^{\prime}[\xi]-\mathscr{A}^{\prime}[0]\right\|_{\mathscr{L}(X, \boldsymbol{Y})}\right) .
$$

Then the equation $\mathscr{A}[\xi]=0$ has a unique solution $\xi$ in an open ball $B_{X}\left(\delta_{1}\right) \subset$ $B_{X}(\delta)$, where $\delta_{1}$ is any number in $(0, \delta)$ for which

$$
\left\|\boldsymbol{f}\left[\varphi_{0}\right]\right\|_{\boldsymbol{Y}}<\delta_{1}\left(\left\|\mathscr{A}^{\prime}[0]^{-1}\right\|_{\mathscr{L}(\boldsymbol{Y}, X)}^{-1}-\sup _{\|\xi\|_{X}<\delta_{1}}\left\|\mathscr{A}^{\prime}[\xi]-\mathscr{A}^{\prime}[0]\right\|_{\mathscr{L}(X, \boldsymbol{Y})}\right) .
$$

Moreover, the mapping $\varphi:=\exp _{\varphi_{0}} \xi$ satisfies the boundary value problem (42)-(43).

(c) Assume further that $\varphi_{0}$ is injective and orientation-preserving. There exists $\varepsilon_{2} \in\left(0, \varepsilon_{1}\right)$ such that, if $\left\|\boldsymbol{f}\left[\varphi_{0}\right]\right\|_{\boldsymbol{Y}}<\varepsilon_{2}$, the deformation $\varphi:=\exp _{\varphi_{0}} \xi$ found in (b) is injective and orientation-preserving.

Proof. (a) It is clear from the discussion preceding the theorem that $\mathscr{A} \in \mathscr{C}^{1}\left(B_{X}(\boldsymbol{\delta}), \boldsymbol{Y}\right)$. Let $\xi \in B_{X}(\delta)$ and let $\varphi:=\exp _{\varphi_{0}} \xi$. We have seen in the Section 6 that 


$$
\operatorname{div} \boldsymbol{T}[\varphi]+\boldsymbol{f}[\varphi]=\operatorname{div}_{0} \boldsymbol{T}^{\mathrm{lin}}[\xi]+\boldsymbol{f}^{\mathrm{aff}}[\xi]+o\left(\|\xi\|_{\mathscr{C}^{1}(T M)}\right),
$$

where div and $\operatorname{div}_{0}$ denote the divergence operators induced by the connections $\nabla:=\nabla[\varphi]$ and $\nabla_{0}:=\nabla\left[\varphi_{0}\right]$, respectively; cf. relations (33) and (34).

Using the definitions of the mappings $f^{\text {aff }}, \mathscr{A}^{\text {lin }}$, and $\mathscr{A}$ (see (27), (41), and (45), respectively) in this relation, we deduce that

$$
\mathscr{A}[\xi]=f\left[\varphi_{0}\right]+\mathscr{A}^{\operatorname{lin}}[\xi]+o\left(\|\xi\|_{\mathscr{C}^{1}(T M)}\right) .
$$

This relation shows that $\mathscr{A}^{\prime}[0]=\mathscr{A}^{\text {lin }}$. Since $\mathscr{A}^{\text {lin }}$ is precisely the differential operator appearing in Theorem 7(b), and since assumption (55) of Theorem 8 is the same as assumption (38) of Theorem 7 when $\Gamma_{2}=\emptyset$, Theorem 7(b) implies that $\mathscr{A}^{\prime}[0] \in \mathscr{L}(X, \boldsymbol{Y})$ is bijective and $\mathscr{A}^{\prime}[0]^{-1} \in \mathscr{L}(\boldsymbol{Y}, X)$.

(b) The idea is to prove that the relations

$$
\xi_{1}:=0 \text { and } \xi_{k+1}:=\xi_{k}-\mathscr{A}^{\prime}[0]^{-1} \mathscr{A}\left[\xi_{k}\right], k \geq 1,
$$

define a convergent sequence in $X$, since then its limit will clearly be a zero of $\mathscr{A}$. This will be done by applying the contraction mapping theorem to the mapping $\mathscr{B}: V \subset B_{X}(\boldsymbol{\delta}) \rightarrow \boldsymbol{Y}$ defined by

$$
\mathscr{B}[\xi]:=\xi-\mathscr{A}^{\prime}[0]^{-1} \mathscr{A}[\xi] .
$$

The set $V$ has to be endowed with a distance that makes $V$ a complete metric space and must be defined in such a way that $\mathscr{B}$ be a contraction and $\mathscr{B}[V] \subset V$.

Since the mapping $\mathscr{A}^{\prime}: B_{X}(\delta) \rightarrow \mathscr{L}(X, \boldsymbol{Y})$ is continuous, it is clear that $\varepsilon_{1}>0$. Hence there exists $\delta_{1} \in(0, \delta)$ such that

$$
\left\|\boldsymbol{f}\left[\varphi_{0}\right]\right\|_{\boldsymbol{Y}}<\delta_{1}\left(\left\|\mathscr{A}^{\prime}[0]^{-1}\right\|_{\mathscr{L}(\boldsymbol{Y}, X)}^{-1}-\sup _{\|\xi\|_{X}<\delta_{1}}\left\|\mathscr{A}^{\prime}[\xi]-\mathscr{A}^{\prime}[0]\right\|_{\mathscr{L}(X, \boldsymbol{Y})}\right) .
$$

Note that this definition is the same as that appearing in the statement of the theorem; cf (57). So pick such a $\delta_{1}$ and define

$$
V=B_{X}\left(\delta_{1}\right]:=\left\{\xi \in X ;\|\xi\|_{X} \leq \delta_{1}\right\}
$$

as the closed ball in $X$ of radius $\delta_{1}$ centered at the origin of $X$. As a closed subspace of the Banach space $\left(X,\|\cdot\|_{X}\right)$, the set $B_{X}\left(\delta_{1}\right]$ endowed with the distance induced by the norm $\|\cdot\|_{X}$ is a complete metric space. Besides, the mapping $\mathscr{B}: B_{X}\left(\delta_{1}\right] \rightarrow X$ is well defined since $B_{X}\left(\delta_{1}\right] \subset B_{X}(\delta)$. It remains to prove that $\mathscr{B}$ is a contraction and that $\mathscr{B}\left[B_{X}\left(\delta_{1}\right]\right] \subset B_{X}\left(\delta_{1}\right]$.

Let $\xi$ and $\eta$ be two elements of $B_{X}\left(\delta_{1}\right]$. Then

$$
\|\mathscr{B}[\xi]-\mathscr{B}[\eta]\|_{X} \leq\left\|\mathscr{A}^{\prime}[0]^{-1}\right\|_{\mathscr{L}(\boldsymbol{Y}, X)}\left\|\mathscr{A}[\eta]-\mathscr{A}[\xi]-\mathscr{A}^{\prime}[0](\xi-\eta)\right\|_{\boldsymbol{Y}}
$$

Applying the mean value theorem to the mapping $\mathscr{A} \in \mathscr{C}^{1}\left(B_{X}(\boldsymbol{\delta}), \boldsymbol{Y}\right)$ next implies that 


$$
\|\mathscr{B}[\xi]-\mathscr{B}[\eta]\|_{X} \leq C_{\mathscr{B}}\|\xi-\eta\|_{X},
$$

where

$$
C_{\mathscr{B}}:=\left\|\mathscr{A}^{\prime}[0]^{-1}\right\|_{\mathscr{L}(\boldsymbol{Y}, X)} \sup _{\|\zeta\|<\delta_{1}}\left\|\mathscr{A}^{\prime}[\zeta]-\mathscr{A}^{\prime}[0]\right\|_{\mathscr{L}(X, \boldsymbol{Y})}
$$

But the inequality (58) implies that

$$
\begin{aligned}
C_{\mathscr{B}} & =1-\left\|\mathscr{A}^{\prime}[0]^{-1}\right\|_{\mathscr{L}(\boldsymbol{Y}, X)}\left(\left\|\mathscr{A}^{\prime}[0]^{-1}\right\|_{\mathscr{L}(\boldsymbol{Y}, X)}^{-1}-\sup _{\|\zeta\|<\delta_{1}}\left\|\mathscr{A}^{\prime}[\zeta]-\mathscr{A}^{\prime}[0]\right\|_{\mathscr{L}(X, \boldsymbol{Y})}\right) \\
& <1-\left\|\mathscr{A}^{\prime}[0]^{-1}\right\|_{\mathscr{L}(\boldsymbol{Y}, X)} \frac{\left\|\boldsymbol{f}\left[\varphi_{0}\right]\right\|_{\boldsymbol{Y}}}{\delta_{1}} \leq 1,
\end{aligned}
$$

which shows that $\mathscr{B}$ is indeed a contraction on $B_{X}\left(\delta_{1}\right]$.

Let $\xi$ be any element of $B_{X}\left(\delta_{1}\right]$. Since

$$
\|\mathscr{B}[\xi]\|_{X} \leq\|\mathscr{B}[0]\|_{X}+\|\mathscr{B}[\xi]-\mathscr{B}[0]\|_{X} \leq\left\|\mathscr{A}^{\prime}[0]^{-1} \boldsymbol{f}\left[\varphi_{0}\right]\right\|_{X}+C_{\mathscr{B}} \delta_{1},
$$

using the above expression of $C_{\mathscr{B}}$ and the inequality (58) yields

$$
\|\mathscr{B}[\xi]\|_{X} \leq\left\|\mathscr{A}^{\prime}[0]^{-1}\right\|_{\mathscr{L}(\boldsymbol{Y}, X)}\left(\left\|\boldsymbol{f}\left[\varphi_{0}\right]\right\|_{\boldsymbol{Y}}+\delta_{1} \sup _{\|\zeta\|<\delta_{1}}\left\|\mathscr{A}^{\prime}[\zeta]-\mathscr{A}^{\prime}[0]\right\|_{\mathscr{L}(X, \boldsymbol{Y})}\right)<\delta_{1},
$$

which shows that $\mathscr{B}\left[B_{X}\left(\delta_{1}\right]\right] \subset B_{X}\left(\delta_{1}\right]$.

The assumptions of the contraction mapping theorem being satisfied by the mapping $\mathscr{B}$, there exists a unique $\xi \in B_{X}\left(\delta_{1}\right]$ such that $\mathscr{B}[\xi]=\xi$, which means that $\xi$ satisfies the equation $\mathscr{A}[\xi]=0$. This equation being equivalent to the boundary value problem (44), the deformation $\varphi:=\exp _{\varphi_{0}} \xi$ satisfies the boundary value problem (42)-(43).

(c) The contraction mapping theorem shows that the rate at which the sequence $\xi_{k}=\mathscr{B}^{k}[0], k=1,2, \ldots$, converges to the solution $\xi$ of the equation $\mathscr{A}[\xi]=0$ satisfies

$$
\left\|\xi_{k}-\xi\right\|_{X} \leq \frac{\left(C_{\mathscr{B}}\right)^{k}}{1-C_{\mathscr{B}}}\|\mathscr{B}[0]\|_{X}
$$

In particular, for $k=0$,

$$
\|\xi\|_{X} \leq \frac{1}{1-C_{\mathscr{B}}}\|\mathscr{B}[0]\| \leq \frac{\left\|\mathscr{A}^{\prime}[0]^{-1}\right\|_{\mathscr{L}(\boldsymbol{Y}, X)}}{1-C_{\mathscr{B}}}\left\|\boldsymbol{f}\left[\varphi_{0}\right]\right\|_{\boldsymbol{Y}} \leq C_{\mathscr{A}}\left\|\boldsymbol{f}\left[\varphi_{0}\right]\right\|_{\boldsymbol{Y}}
$$

where

$$
C_{\mathscr{A}}:=\left\{\left\|\mathscr{A}^{\prime}[0]^{-1}\right\|_{\mathscr{L}(\boldsymbol{Y}, X)}^{-1}-\sup _{\|\zeta\|<\delta_{1}}\left\|\mathscr{A}^{\prime}[\zeta]-\mathscr{A}^{\prime}[0]\right\|_{\mathscr{L}(X, \boldsymbol{Y})}\right\}^{-1}
$$

The Sobolev embedding $W^{m+2, p}(T M) \subset C^{1}(T M)$ being continuous, the mapping

$$
\eta \in B_{X}\left(\delta_{1}\right] \rightarrow \psi:=\exp _{\varphi_{0}} \eta \in \mathscr{C}^{1}(M, N) \rightarrow \operatorname{det}(D \psi) \in \mathscr{C}^{0}(M)
$$


is also continuous. Besides $\min _{z \in M} \operatorname{det}\left(D \varphi_{0}(z)\right)>0$ since $\varphi_{0}$ is orientation-preserving and $M$ is compact. It follows that there exists $0<\delta_{2} \leq \delta_{1}$ such that

$$
\|\eta\|_{X}<\delta_{2} \Rightarrow\left\|\operatorname{det}(D \psi)-\operatorname{det}\left(D \varphi_{0}\right)\right\|_{\mathscr{C}^{0}(M)}<\min _{z \in M} \operatorname{det}\left(D \varphi_{0}(z)\right)
$$

which next implies that

$$
\|\eta\|_{X}<\delta_{2} \Rightarrow \operatorname{det}(D \psi(x))>0 \text { for all } x \in M
$$

Assume now that the applied forces satisfy $\left\|f\left[\varphi_{0}\right]\right\|_{Y}<\varepsilon_{2}:=\delta_{2} / C_{\mathscr{A}}$. Then the relations (59) and (60) together show that the deformation $\varphi:=\exp _{\varphi_{0}} \xi$, where $\xi \in$ $B_{X}\left(\delta_{1}\right]$ denotes the solution of the equation $\mathscr{A}[\xi]=0$, satisfies

$$
\operatorname{det}(D \varphi(x))>0 \text { for all } x \in M,
$$

which means that $\varphi$ is orientation-preserving.

Moreover, since $\varphi=\varphi_{0}$ on $\partial M$ and $\varphi_{0}: M \rightarrow N$ is injective, the inequality $\operatorname{det} D \varphi(x)>0$ for all $x \in M$ implies that $\varphi: M \rightarrow N$ is injective; cf. Ciarlet [10, Theorem 5.5-2].

Remark 7. (a) The mapping $\mathscr{F}: B_{X}(\delta) \subset X \rightarrow \boldsymbol{Y}$ defined by

$$
\mathscr{F}[\xi]:=\mathscr{A}[\xi]-f\left[\varphi_{0}\right]
$$

satisfies the assumptions of the local inversion theorem at the origin of $X$ if the assumption (55) is satisfied. Hence there exist constants $\delta_{3}>0$ and $\varepsilon_{3}>0$ such that the equation $\mathscr{F}[\xi]=-\boldsymbol{f}\left[\varphi_{0}\right]$, or equivalently

$$
\mathscr{A}[\xi]=0,
$$

has a unique solution $\xi \in X,\|\xi\|_{X}<\delta_{3}$, if $\left\|f\left[\varphi_{0}\right]\right\|_{\boldsymbol{Y}}<\varepsilon_{3}$. Using the Banach contraction theorem instead of the local inversion theorem in the proof of Theorem 7 provides (as expected) explicit estimations of the constants $\delta_{3}$ and $\varepsilon_{3}$, namely $\delta_{3}=\delta_{1}$ and $\varepsilon_{3}=\varepsilon_{1}$ (see (56) and (57) for the definitions of $\varepsilon_{1}$ and $\delta_{1}$ ).

(b) Previous existence theorems for the equations of nonlinear elasticity in Euclidean spaces (see, e.g., Ciarlet [10] and Valent [26]) can be obtained from Theorem 8 by making additional assumptions on the applied forces: either $\dddot{\boldsymbol{f}}-\boldsymbol{f}\left[\varphi_{0}\right]=0$ in the case of "dead" forces, or $\ddot{f} \in \mathscr{C}^{m}\left(M \times T M \times T_{1}^{1} M, T^{*} M \otimes \boldsymbol{\Lambda}^{n} M\right)$ in the case of "live" forces.

(c) Theorem $7(a)$ and $(b)$ can be generalized to mixed Dirichlet-Neumann boundary conditions provided that $\bar{\Gamma}_{1} \cap \bar{\Gamma}_{2}=\emptyset$, since in that case the regularity theorem for elliptic systems of partial differential equations still holds. 


\section{References}

1. R. Abraham, J.E. Marsden and T. Ratiu, Manifolds, Tensor Analysis, and Applications, Springer Verlag, New York, 1988.

2. C. Amrouche and V. Girault, Decomposition of vector spaces and application to the Stokes problem in arbitrary dimension, Czech. Math. J. 44 (1994) 109-140.

3. L. Andersson, R. Beig and B.G. Schmidt, Static self-gravitating elastic bodies in Einstein gravity, Communications on Pure and Applied Mathematics LXI (2008), 0988-1023.

4. T. Aubin, Some Nonlinear Problems in Riemannian Geometry, Springer, Berlin, 2010.

5. R. Beig and B.G. Schmidt, Relativistic elasticity. Classical Quantum Gravity 20 (2003), 889904.

6. R. Beig and B.G. Schmidt, Static, self-gravitating elastic bodies, R. Soc. Lond. Proc. A 459 (2003), 109-115.

7. R. Beig and B.G. Schmidt, Relativistic elastostatics. I. Bodies in rigid rotation. Classical Quantum Gravity 22 (2005), 2249-2268.

8. R. Beig and M. Wernig-Pichler, On the motion of a compact elastic body Commun. Math. Phys. 271 (2007), 455-465.

9. B. Carter and H. Quintana, Foundations of General Relativistic High-Pressure Elasticity Theory, Proc. R. Soc. Lond. A 331 (1972), 57-83.

10. P.G. Ciarlet, Mathematical Elasticity, Volume I: Three-Dimensional Elasticity, North-Holland, Amsterdam, 1988

11. P.G. Ciarlet, An Introduction to Differential Geometry with Applications to Elasticity, Springer, Dordrecht, 2005.

12. P.G. Ciarlet and C. Mardare, On the Newton-Kantorovich theorem, Analysis and Applications, Anal. Appl. 10 (2012), 249-269.

13. G. Duvaut and J.L. Lions, Inequalities in Mechanics and Physics, Springer, 1978.

14. W. Chen and J. Jost, A Riemannian version of Korn's inequality, Calc. Var. 14 (2002), 517530.

15. E. Efrati, E. Sharon and R. Kupferman, Elastic theory of unconstrained non-Euclidean plates, Journal of the Mechanics and Physics of Solids 57 (2009), 762-775.

16. M. Epstein and R. Segev, Differentiable manifolds and the principle of virtual work in continuum mechanics, J. Math. Phys. 21 (1980), 1243-1245.

17. Grubic N., LeFloch P.G. and Mardare C., Mathematical elasticity theory in a Riemannian manifold, arXiv:1312.3599 [math.AP].

18. V.A. Kondrat'ev and O.A. Oleinik, On the dependence of the constant in Korn's inequality on parameters characterising the geometry of the region, Uspekhi Mat. Nauk 44 (1989), 153-160. English translation: Russian Math. Surveys 44 (1989), 187-195.

19. J.E. Marsden and T.J.R. Hughes, Mathematical Foundations of Elasticity, Prentice-Hall, New Jersey, 1983.

20. K. Nomizu, On local and global existence of Killing vector fields, Annals of Mathematics 72 (1960), 105-120.

21. R. Segev, Forces and the existence of stresses in invariant continuum mechanics. J. Math. Phys. 27 (1986), 163-170.

22. R. Segev and G. Rodnay, Cauchy's theorem on manifolds. J. Elasticity 56 (1999), 129-144.

23. R. Segev, The geometry of Cauchy's fluxes. Arch. Ration. Mech. Anal.154 (2000), 183-198.

24. R. Segev, A correction of an inconsistency in my paper: "Cauchy's theorem on manifolds", $J$. Elasticity 63 (2001), 55-59.

25. H.C. Simpson and S.J. Spector, Applications of estimates near the boundary to regularity of solutions in linearized elasticity, SIAM J. Math. Anal. 41 (2009), 923-935.

26. T. Valent, Boundary Value Problems of Finite Elasticity: Local Theorems on Existence, Uniqueness, and Analytic Dependence on Data Springer, New York, 1988.

27. M. Wernig-Pichler, Relativistic Elastodynamics, arXiv:gr-qc/0605025v1, 2006. 